\title{
Robust Optimal Attitude Controller for MIMO Uncertain Hexarotor MAVs: Disturbance Observer-Based
}

\author{
Nurul Dayana Salim, ${ }^{1,2}$ Dafizal Derawi, ${ }^{1,2}$ Hairi Zamzuri, ${ }^{1}$ \\ Kenzo Nonami, ${ }^{3,4}$ and Mohd Azizi Abdul Rahman ${ }^{1}$ \\ ${ }^{1}$ Malaysia-Japan International Institute of Technology, Universiti Teknologi Malaysia, 54100 Kuala Lumpur, Malaysia \\ ${ }^{2}$ Mechatronic Systems Laboratory, Robotic Systems Enterprise, 75250 Melaka, Malaysia \\ ${ }^{3}$ Department of Mechanical Engineering, Graduate School of Engineering, Chiba University, Chiba Prefecture, \\ Chiba 263-0022, Japan \\ ${ }^{4}$ Autonomous Control Systems Laboratory Ltd., Chiba-ken, Chiba 263-8522, Japan
}

Correspondence should be addressed to Nurul Dayana Salim; nuruldayanasalim@yahoo.com

Received 22 October 2015; Accepted 31 March 2016

Academic Editor: Andrzej Swierniak

Copyright ( 2016 Nurul Dayana Salim et al. This is an open access article distributed under the Creative Commons Attribution License, which permits unrestricted use, distribution, and reproduction in any medium, provided the original work is properly cited.

\begin{abstract}
This paper proposes a robust optimal attitude control design for multiple-input, multiple-output (MIMO) uncertain hexarotor micro aerial vehicles (MAVs) in the presence of parametric uncertainties, external time-varying disturbances, nonlinear dynamics, and coupling. The parametric uncertainties, external time-varying disturbances, nonlinear dynamics, and coupling are treated as the total disturbance in the proposed design. The proposed controller is achieved in two simple steps. First, an optimal linearquadratic regulator (LQR) controller is designed to guarantee that the nominal closed-loop system is asymptotically stable without considering the total disturbance. After that, a disturbance observer is integrated into the closed-loop system to estimate the total disturbance acting on the system. The total disturbance is compensated by a compensation input based on the estimated total disturbance. Robust properties analysis is given to prove that the state is ultimately bounded in specified boundaries. Simulation results illustrate the robustness of the disturbance observer-based optimal attitude control design for hovering and aggressive flight missions in the presence of the total disturbance.
\end{abstract}

\section{Introduction}

In recent years, a great deal of research has been conducted on the control of multirotor micro aerial vehicles (MAVs) around the world as their ability to perform certain task that are unable or difficult to complete by humans. The interest for these aerial vehicles is growing in civilian and military applications such as search and rescue mission, inspection (for wind turbines, power lines, pipelines, and etc.), surveillance, security, and agriculture. For most recent applications, multirotor MAVs have been used to create damage assessment maps for disaster efforts. In contrast to traditional helicopters and fixed wing aerial vehicles, multirotor MAVs are much safer to operate, are highly manoeuvrable, can easily hover above target, are able to do vertical takeoff and landing, are able to fly at low altitude, and are a low cost solution. However, the multirotor MAV is an underactuated system because it has six degrees of freedom but only four control inputs are available. Thus, the autonomous flight control design of multirotor MAV is a challenging task.

Quadrotor is the most popular multirotor MAVs among researchers in the previous studies. Based on reported results, the quadrotor MAVs have successfully achieved their stability in hovering condition by the proportional-integral-derivative (PID) control method $[1,2]$. In general, the PID control design is straightforward and can easily be implemented in a real-time embedded system. However, the performances of the PID controller will be degraded under the effects of nonlinear dynamics and coupling. Therefore, many modelbased control methods have been extensively studied to restrain the influence of nonlinearities and coupling in dynamic model of multirotor MAVs such as a flatness-based 
nonlinear control method [3], a dynamic inversion control method [4], a hierarchical control method [5], a nonlinear control method using nested saturations [6], a backsteppingbased control method [7], a model predictive controller (MPC) [8], a quaternion-based nonlinear feedback controller [9], an optimal LQR controller [10], and a backstepping-based inverse optimal controller [11]. Actually, those controllers require an accurate model of hexarotor in order to restrain the effects of nonlinear dynamics and coupling. Based on the accurate model, the steady-state and dynamical tracking performances can be achieved. However, the effects of parametric uncertainties and external disturbances were not fully covered in those model-based controllers. In reality, an exact mathematical model of a complex dynamic system like the multirotor MAVs cannot be obtained because the model is only an approximation of the real system. The multirotor MAVs are small and lightweight vehicles and, thus, it is easier to be affected by the payload mass variation. Furthermore, the exact vehicle parameters are difficult to be measured or obtained and normally regarded as constants thus subject to parametric uncertainties. In practice, the closed-loop system can be affected by the external disturbances (e.g., wind disturbances) and instability may happen.

Therefore, many flight control methods have been proposed in literature to encounter the effect of parametric uncertainties and external disturbances. In previous studies, many researches focused on parametric uncertainties problems only such as an adaptive command-filtered backstepping controller [12], a model reference adaptive controller [13], an adaptive dynamic feedback-linearization controller [14], and a nonlinear adaptive controller [15]. However, those controllers are not suitable for practical applications in outdoor environments since the closed-loop system will be affected by the external wind disturbances. The new approaches of adaptive scheme called robust adaptive control have been proposed in the presence of modelling errors and external disturbances [16-20]. The proposed method in $[16,18]$ does not require a priori knowledge of the modelling errors and external disturbances. However, the implementation issues of adaptive law need to be addressed. In [17], the proposed method introduced a new approach using robust integral of the signum of the error (RISE) and immersion and invariance- (I\&I-) based adaptive control method to compensate the modelling errors and external disturbances. The proposed method in [20] has considered the presence of constant wind disturbances only. In general, those adaptive controllers could achieve the robust asymptotically tracking performances for the closed-loop system, but the desired robust properties of the controller cannot be specified. Based on sliding mode control (SMC) method, the new developed and higher order SMC schemes were proposed in [21-24] to deal with parametric uncertainties and external disturbances. In [21], the SMC is driven by sliding mode disturbance observer to provide robustness against external disturbances and uncertainties while [24] has proposed a second-order sliding mode observer to reconstruct disturbances. In [22, 23], the use of higher order sliding mode control and adequate controller tuning was developed to decrease the chattering phenomenon encountered with SMC approach.

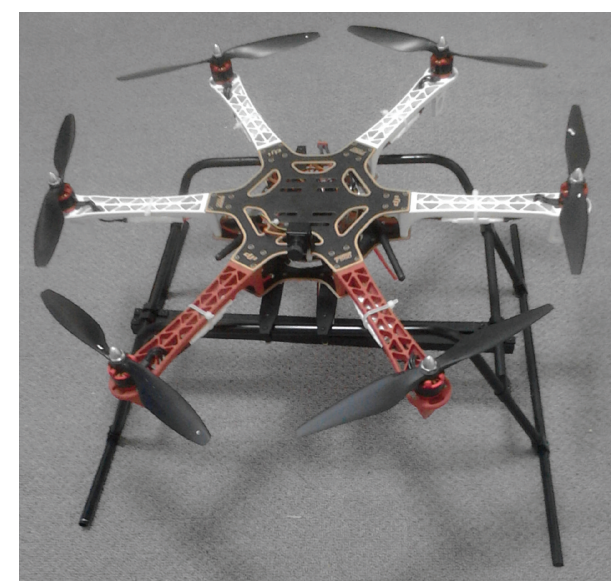

FIGURE 1: The hexarotor research platform.

However, their algorithms are very complicated and difficult to implement. The attitude control problem of quadrotor subject to time-varying disturbances was considered in [25] by combining an extended observer to estimate disturbances and a feedback controller with sliding mode term for stabilization. But the effects of parametric uncertainties were neglected.

In this paper, a new robust optimal attitude control design for multiple-input multiple-output (MIMO) uncertain hexarotor micro aerial vehicles (MAVs) in the presence of parametric uncertainties, external time-varying disturbances, nonlinear dynamics, and coupling is proposed. The parametric uncertainties, external time-varying disturbances, nonlinear dynamics, and coupling are treated as the total disturbance in the proposed design. The proposed controller is achieved in two simple steps. First, an optimal linear-quadratic regulator (LQR) controller is designed to guarantee that the nominal closed-loop system is asymptotically stable without considering the total disturbance. After that, a disturbance observer based on $[26,27]$ is integrated into the closed-loop system to estimate the total disturbance in the system. Then, the total disturbance is compensated from the system by the compensation input based on the estimated total disturbance. Robust properties analysis is given to prove that the state is ultimately bounded in specified boundaries. The simulation results are presented to demonstrate the robustness of the proposed control design for hovering and aggressive flight missions in the presence of the total disturbance, to prove that the state is ultimately bounded in specified boundaries and to demonstrate excellent steadystate and dynamic tracking performances of the closed-loop system.

Compared to previous studies in $[1-6,8-23,25]$, this paper focuses on another potential multirotor MAV platform called hexarotor which has better stability and fault tolerance as shown in Figure 1. Two additional rotors can increase payload, reliability, and manoeuvrability compared to quadrotor because at least four rotors will contribute to each angular rotation. Therefore, the forces and torques generated by two additional rotors will result in different torques about each 


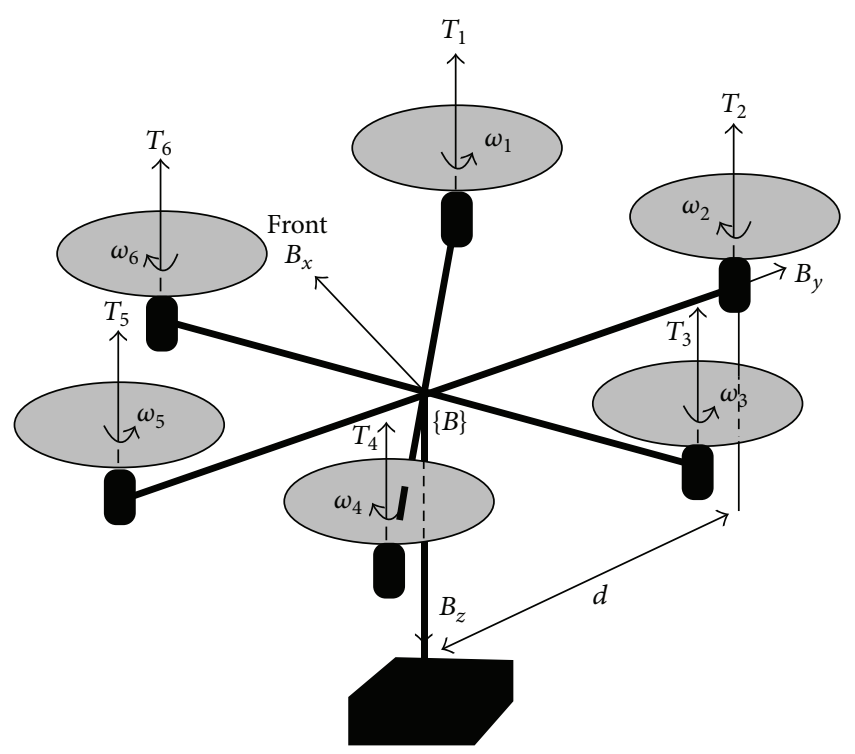

FIgURE 2: The schematic of hexarotor. The hexarotor is attached on the spherical joint without translational motions.

axis and thus affect the dynamical response. Compared to several researches on hexarotor [28-30], the present paper treats hexarotor MAV as the MIMO system rather than the combination of multiple single-input single-output (SISO) systems. This paper also demonstrates the effectiveness of the proposed method for aggressive manoeuvres under the effects of multiple uncertainties. Compared to [3-11], the proposed controller does not require an accurate model and is able to compensate the total disturbance. In addition, the proposed controller can improve the steady-state and dynamic attitude tracking performances by introducing the disturbance observer to estimate the total disturbance acting on the system. Compared to $[16-20,29,30]$, the desired robust properties can be specified by the proposed controller where the nominal responses can be specified by the optimal LQR controller and the effects of the total disturbance can be compensated by the compensation input based on the estimated total disturbance.

This paper is structured as follows. The model of a hexarotor is described in Section 2. Section 3 presents the proposed robust optimal attitude control design. Then, the robust properties are analysed and are proven in Section 4. After this, the simulation results are presented and discussed. Finally, this paper is summarized in Section 6.

\section{Model of Hexarotor}

The hexarotor is a rigid body that consists of six rotors to generate the required forces and torques as shown in Figure 1. Let $\left\{B_{x}, B_{y}, B_{z}\right\}$ denote unit vectors along the respective body-fixed frame expressed in $\{B\}$ for the hexarotor airframe as presented in Figure 2. Let $\eta(t)=\left(\begin{array}{lll}\phi(t) & \theta(t) & \psi(t)\end{array}\right)^{T}$ denote the attitude that represents roll $\phi(t)$, pitch $\theta(t)$, and yaw $\psi(t)$ angles. Then, the orientation of hexarotor is given by a rotation matrix in the special orthogonal group $R(t) \in \mathrm{SO}(3)$ using $z-y-x$ Euler angles convention, expressed as

$$
R(t)=\left(\begin{array}{ccc}
c \theta(t) c \psi(t) & \mathrm{s} \phi(t) \mathrm{s} \theta(t) \mathrm{c} \psi(t)-c \phi(t) \mathrm{s} \psi(t) & \mathrm{c} \phi(t) \mathrm{s} \theta(t) \mathrm{c} \psi(t)+\mathrm{s} \phi(t) \mathrm{s} \psi(t) \\
\mathrm{c} \theta(t) \mathrm{s} \psi(t) & \mathrm{s} \phi(t) \mathrm{s} \theta(t) \mathrm{s} \psi(t)+c \phi(t) \mathrm{c} \psi(t) & \mathrm{c} \phi(t) \mathrm{s} \theta(t) \mathrm{s} \psi(t)-\mathrm{s} \phi(t) \mathrm{c} \psi(t) \\
-\mathrm{s} \theta(t) & \mathrm{s} \phi(t) \mathrm{c} \theta(t) & \mathrm{c} \phi(t) \mathrm{c} \theta(t)
\end{array}\right)
$$

where $\mathrm{c}$ and $\mathrm{s}$ denote cosine and sine, respectively. Let $\Omega(t)=$ $\left(\Omega_{x}(t) \Omega_{y}(t) \Omega_{z}(t)\right)^{T} \in \mathbb{R}^{3}$ denote the angular velocity expressed in $\{B\}$ and the standard attitude kinematics are given by

$$
\dot{R}(t)=R(t) \Omega_{\times}(t),
$$

where $\Omega_{\times}(t)$ represents the skew-symmetric matrix, such that $\Omega_{\times}(t) a=\Omega(t) \times a$ for vector cross product $\times$ and any vector $a \in \mathbb{R}^{3}$, where

$$
\Omega_{\times}(t)=\left(\begin{array}{ccc}
0 & -\Omega_{z}(t) & \Omega_{y}(t) \\
\Omega_{z}(t) & 0 & -\Omega_{x}(t) \\
-\Omega_{y}(t) & \Omega_{x}(t) & 0
\end{array}\right)
$$

As described in [31], the mathematical model of angular dynamics of hexarotor can be derived as

$$
J \dot{\Omega}(t)+\Omega_{\times}(t) J \Omega(t)=\tau(t)+w(t),
$$

where $J \in \mathbb{R}^{3 \times 3}$ denote the inertia matrix, $\tau(t)=$ $\left(\tau_{\phi}(t) \tau_{\theta}(t) \tau_{\psi}(t)\right)^{T} \in \mathbb{R}^{3}$ denote the torque applied to the airframe in $\{B\}$ by the aerodynamics of the rotors, and $w(t)=$ $\left(w_{\phi}(t) w_{\theta}(t) w_{\psi}(t)\right)^{T} \in \mathbb{R}^{3}$ denote the external timevarying disturbance (e.g., wind disturbances). The hexarotor's mass distribution is assumed to be symmetrical with respect to coordinate frame and thus the products of inertia in $J$ are zero. Therefore, $J$ becomes a diagonal matrix that consists of moments of inertia, $J=\operatorname{diag}\left(J_{x x}, J_{y y}, J_{z z}\right)$.

Let $T_{i}(t)(i=1,2, \ldots, 6)$ denote the hover thrust force generated by individual rotor and it can be expressed as [31]

$$
T_{i}(t)=b \omega_{i}^{2}(t),
$$

where $b$ is the positive lift constant that can be obtained from static thrust tests and it depends on the chord length of the blade, the number of blades, the blade radius, and the air density. $\omega_{i}(t)(i=1,2, \ldots, 6)$ is the angular velocity of the rotors. Based on [31], there exist the secondary aerodynamic forces on the translational dynamics when 
the rotor is not in hover. However, these effects can be ignored for attitude control design which depends on angular dynamics of hexarotor only.

The reaction torque $Q_{i}(t)(i=1,2, \ldots, 6)$ generated by each hovering rotor can be modelled as [31]

$$
Q_{i}(t)=k \omega_{i}^{2}(t)
$$

where $k$ is a positive torque constant that has relation with $b$ and it can be determined by static thrust tests as well.

The total thrust force $T(t)$ for hovering is the sum of thrusts from each rotor:

$$
T(t)=\sum_{i=1}^{6} T_{i}(t)
$$

and its control input $u_{T}(t)$ to compensate the gravitational force can be defined as

$$
\begin{aligned}
u_{T}(t)= & \omega_{1}^{2}(t)+\omega_{2}^{2}(t)+{\omega_{3}}^{2}(t)+\omega_{4}^{2}(t)+\omega_{5}^{2}(t) \\
& +\omega_{6}^{2}(t) .
\end{aligned}
$$

The torque $\tau$ can be expressed as

$$
\begin{aligned}
& \tau(t)=\left(\begin{array}{c}
\tau_{\phi}(t) \\
\tau_{\theta}(t) \\
\tau_{\psi}(t)
\end{array}\right) \\
& =\left(\begin{array}{c}
d\left(T_{5}(t)-T_{2}(t)\right)+\frac{\left(T_{6}(t)+T_{4}(t)-T_{3}(t)-T_{1}(t)\right) d}{2} \\
\frac{\left(T_{1}(t)+T_{6}(t)-T_{4}(t)-T_{3}(t)\right) d \sqrt{3}}{2} \\
Q_{1}(t)-Q_{2}(t)+Q_{3}(t)-Q_{4}(t)+Q_{5}(t)-Q_{6}(t)
\end{array}\right),
\end{aligned}
$$

where $d$ is the distance from the centre of the rotor to the centre of mass.

The control input of attitude system $u(t)=$ $\left(u_{\phi}(t) u_{\theta}(t) \quad u_{\psi}(t)\right)^{T} \in \mathbb{R}^{3}$ can be defined as

$$
u(t)=\left(\begin{array}{c}
u_{\phi}(t) \\
u_{\theta}(t) \\
u_{\psi}(t)
\end{array}\right)=\left(\begin{array}{c}
\left(\omega_{5}^{2}(t)-\omega_{2}^{2}(t)\right)+\frac{\left(\omega_{6}^{2}(t)+\omega_{4}^{2}(t)-\omega_{3}^{2}(t)-\omega_{1}^{2}(t)\right)}{2} \\
\frac{\left(\omega_{1}^{2}(t)+\omega_{6}^{2}(t)-\omega_{4}^{2}(t)-\omega_{3}^{2}(t)\right) \sqrt{3}}{2} \\
\omega_{1}^{2}(t)-\omega_{2}^{2}(t)+\omega_{3}^{2}(t)-\omega_{4}^{2}(t)+\omega_{5}^{2}(t)-\omega_{6}^{2}(t)
\end{array}\right) .
$$

Actually, the motion of hexarotor can be controlled directly using control input $u(t)$ and $u_{T}(t)$ because, in practice, the values of $u(t)$ and $u_{T}(t)$ will be combined through control mixer and then will be transformed to pulse width modulation values (PWM) (based on embedded flight controller specifications) with certain limit (upper and lower saturation values). Then, the PWM values will be distributed to each rotor by an electronic speed controller (ESC). Based on (4), (9), and (10), the attitude control input $u(t)$ is proportional to torque $\tau(t)$ such that $J^{-1} \tau(t)=V u(t)$, where vehicle parameter matrix $V \in \mathbb{R}^{3 \times 3}$ can be defined as

$$
V=\left(\begin{array}{ccc}
\frac{d b}{J_{x x}} & 0 & 0 \\
0 & \frac{d b}{J_{y y}} & 0 \\
0 & 0 & \frac{k}{J_{z z}}
\end{array}\right)
$$

The vehicle parameter $V$ is a combination of nominal $(N)$ and uncertain $(\Delta)$ parts as

$$
V=V^{N}+V^{\Delta}
$$

Finally, the hexarotor model in (4) can be rewritten as

$$
\begin{aligned}
\dot{\Omega}(t)= & -J^{-1} \Omega_{\times}(t) J \Omega(t)+V^{N} u(t)+V^{\Delta} u(t) \\
& +J^{-1} w(t) .
\end{aligned}
$$

Based on (13), let us compute the tracking error model of hexarotor as

$$
\dot{\Omega}(t)-\dot{\Omega}^{r}(t)=V^{N}(u(t)+\Delta(t)),
$$

where $\dot{\Omega}^{r}(t)$ is the desired angular acceleration in $\{B\}$ and $\Delta(t)=\left(\Delta_{\phi}(t) \quad \Delta_{\theta}(t) \quad \Delta_{\psi}(t)\right)^{T} \in \mathbb{R}^{3}$ is the total disturbance which consists of multiple uncertainties with the following form:

$$
\begin{aligned}
& \Delta(t)=V^{N^{-1}}\left(-J^{-1} \Omega_{\times}(t) J \Omega(t)+V^{\Delta} u(t)+J^{-1} w(t)\right. \\
& \left.-\dot{\Omega}^{r}(t)\right) .
\end{aligned}
$$

Assumption 1. The uncertain part $V^{\Delta}$ is bounded. The nominal part $V^{N}=\operatorname{diag}\left(V_{\phi}^{N}, V_{\theta}^{N}, V_{\psi}^{N}\right)$ has positive values and satisfies $V^{\Delta}<V^{N}$.

Assumption 2. The pitch angle satisfies $\theta(t) \in(-\pi / 2+$ $\left.\delta_{\theta}, \pi / 2-\delta_{\theta}\right)$, where $\delta_{\theta}>0$.

Assumption 3. The external time-varying disturbance $w(t)$ is bounded.

Assumption 4. The output of attitude system is attitude $\eta(t)$ and the reference signal is desired attitude denoted by $r(t)=$ $\left(r_{\phi}(t) r_{\theta}(t) r_{\psi}(t)\right)^{T} \in \mathbb{R}^{3}$. The reference signals and its 
derivatives $r(t)^{(k)}(k=0,1,2)$ are piecewise uniformly bounded.

Remark 5. There is a singularity when pitch angle $|\theta(t)|=$ $\pi / 2$. Therefore, it is required to avoid $|\theta(t)|=\pi / 2$.

Remark 6. Equation (15) consists of parametric uncertainties, external time-varying disturbances, nonlinear dynamics, and coupling.

\section{Robust Optimal Control Design}

Let us denote $x(t)=\left(x_{1}(t) x_{2}(t) x_{3}(t)\right)^{T} \in \mathbb{R}^{9}$ as state vector, where $x_{1}(t)=\eta(t)-r(t), x_{2}(t)=\dot{x}_{1}(t)$, and $\dot{x}_{3}=$ $x_{1}(t)$. Actually, $x_{1}(t), x_{2}(t)$, and $x_{3}(t)$ are the attitude tracking error, the rate of change in the attitude tracking error (angular velocity tracking error), and the sum of the previous attitude tracking errors, respectively. As can be seen, the tracking error will be managed in three parts, to handle the present, through $x_{1}(t)$, to anticipate the future, through $x_{2}(t)$, and to recover from the past, through $x_{3}(t)$.

Let us define

$$
\begin{aligned}
A & =\left(\begin{array}{lll}
O_{3} & I_{3} & O_{3} \\
O_{3} & O_{3} & O_{3} \\
I_{3} & O_{3} & O_{3}
\end{array}\right)_{9 \times 9}, \\
B & =\left(\begin{array}{c}
O_{3} \\
V^{N} \\
O_{3}
\end{array}\right)_{9 \times 3},
\end{aligned}
$$

where $I_{3}$ and $O_{3}$ are a $3 \times 3$ identity matrix and a $3 \times 3$ zero matrix, respectively.

From (14), the state equation is

$$
\dot{x}(t)=A x(t)+B(u(t)+\Delta(t)) .
$$

The proposed controller consists of the optimal LQR controller and the disturbance observer as depicted in Figure 3. The attitude control input $u(t)$ can be defined as

$$
u(t)=u^{\mathrm{LQR}}(t)+u^{\mathrm{dist}}(t)
$$

where $u^{\mathrm{LQR}}(t)=\left(u_{\phi}{ }^{\mathrm{LQR}}(t) u_{\theta}{ }^{\mathrm{LQR}}(t) u_{\psi}{ }^{\mathrm{LQR}}(t)\right)^{T}$ is the control input computed by the optimal LQR controller and $u^{\text {dist }}(t)=\left(u_{\phi}{ }^{\text {dist }}(t) u_{\theta}{ }^{\text {dist }}(t) \quad u_{\psi}{ }^{\text {dist }}(t)\right)^{T}$ is the compensation input derived based on the estimated total disturbance. The proposed method can be achieved in two simple steps. First, the optimal LQR controller is designed for the nominal system without considering the total disturbance $\Delta(t)$. After that, the disturbance observer is designed and integrated to the closed-loop system to estimate the total disturbance which consists of parametric uncertainties, external disturbances, nonlinear dynamics, and coupling. Then, the total disturbance is compensated from the system by the compensation input in (18).

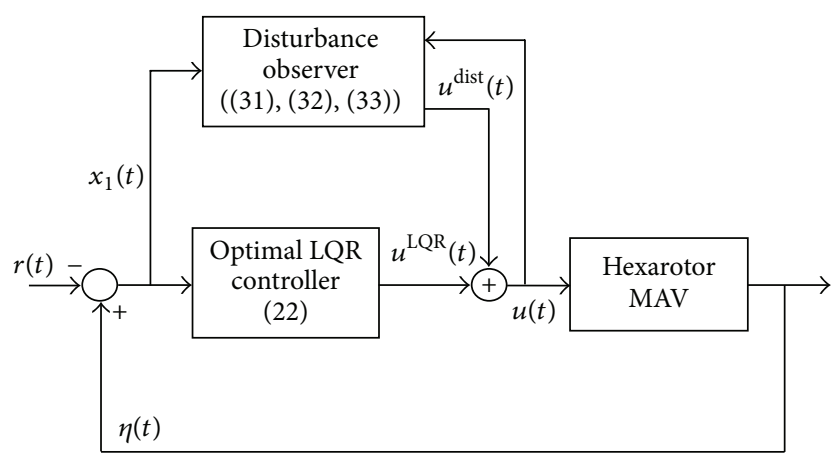

FIGURE 3: The structure of the proposed robust optimal attitude controller for MIMO uncertain hexarotor MAV.

3.1. Step 1: Optimal LQR Control Design. First, the optimal controller is designed based on the LQR control approach for the nominal system without considering the total disturbance $\Delta(t)$ :

$$
\dot{x}(t)=A x(t)+B u(t) .
$$

The quadratic cost functions $\mathscr{f}$ are minimized:

$$
\mathscr{J}=\int_{0}^{\infty}\left(x^{T}(t) Q x(t)+u^{T}(t) r u(t)\right) d t,
$$

where $Q$ is a positive-definite and symmetric matrix and $r$ is a weighting matrix. Let us solve the Riccati equation to return the positive-definite matrices $S$ :

$$
A^{T} S+S A-S B R^{-1} B^{T} S+Q=0 .
$$

Therefore, the linear-quadratic controller is the unique, optimal, full state feedback law:

$$
u^{\mathrm{LQR}}(t)=-K x(t)
$$

with

$$
K=r^{-1} B^{T} S .
$$

Let us substitute (22) into (19); then,

$$
\dot{x}(t)=(A-B K) x(t) .
$$

The closed-loop dynamics are guaranteed to be asymptotically stable if the state $x(t)$ is available for feedback, $\left[\begin{array}{ll}A & B\end{array}\right]$ is stabilizable, and $r=r^{T}>0$.

3.2. Step 2: Disturbance Observer. The system which consists of the total disturbance $\Delta(t)$ is considered by substituting (22) and (18) into (17); one can have

$$
\dot{x}(t)=A_{H} x(t)+B\left(u^{\text {dist }}(t)+\Delta(t)\right),
$$

where $A_{H}=A-B K$ is Hurwitz. The performance of the optimal LQR controller in previous step will be degraded when the hexarotor system is subject to the total disturbance $\Delta(t)$ especially from external time-varying disturbances 
(e.g., wind disturbances in outdoor environments). In this section, the disturbance observer is designed based on [26, 27] in order to estimate the total disturbance acting on the system and then eliminate it in (18).

In practice, the actual value of total disturbance $\Delta(t)$ cannot be determined. From (17), $\Delta(t)$ can be derived as

$$
\Delta(t)=V^{N^{-1}} \ddot{x}_{1}(t)-u(t) .
$$

Equation (26) can be rewritten in terms of $s$ domain as

$$
\Delta(s)=V^{N^{-1}} s^{2} x_{1}(s)-u(s)
$$

where $s$ is the Laplace operator.

Then, both sides of (27) are multiplied by a low-pass filter matrix $G(s)$ in order to estimate the total disturbance:

$$
G(s) \Delta(s)=G(s) V^{N^{-1}} s^{2} x_{1}(s)-G(s) u(s),
$$

where the low-pass filter matrix $G(s)$ is defined to be

$$
G(s)=\left(\begin{array}{ccc}
\frac{g_{\phi}{ }^{2}}{\left(s+g_{\phi}\right)^{2}} & 0 & 0 \\
0 & \frac{g_{\theta}{ }^{2}}{\left(s+g_{\theta}\right)^{2}} & 0 \\
0 & 0 & \frac{g_{\psi}{ }^{2}}{\left(s+g_{\psi}\right)^{2}}
\end{array}\right) \text {. }
$$

Let us define the term $G(s) \Delta(s)$ in (28) as estimated total disturbance $\widehat{\Delta}(s)$. Based on $[26,27]$, if positive constants $g_{\phi}$, $g_{\theta}$, and $g_{\psi}$ are sufficiently large, the filter will have sufficient wide frequency bandwidths. Therefore, the estimated total disturbance is almost equal to actual total disturbance $(\widehat{\Delta}(s) \approx$ $\Delta(s))$.

Let us define the compensation input $u^{\text {dist }}(s)$ as

$$
u^{\text {dist }}(s)=-\widehat{\Delta}(s)
$$

However, the compensation input $u^{\text {dist }}(s)$ in (30) is not suitable for practical implementation because it is in $s$ domain. Therefore, from (28) and (30), the compensation input $u^{\text {dist }}(t)$ can be derived mathematically by introducing two new states $q_{1}$ and $q_{2}$ :

$$
\begin{gathered}
\dot{q}_{1}(t)=A_{q 1} q_{1}(t)+B_{q 1} u_{q 1}(t), \\
\dot{q}_{2}(t)=A_{q 2} q_{2}(t)+B_{q 2} u_{q 2}(t), \\
u^{\text {dist }}(t)=\lambda V^{N^{-1}}\left(x_{1}(t)-q_{2}(t)\right),
\end{gathered}
$$

where $q_{1}(t)=\left(q_{1 \phi}(t) q_{1 \theta}(t) q_{1 \psi}(t)\right)^{T} \in \mathbb{R}^{3}, q_{2}(t)=$ $\left(q_{2 \phi}(t) q_{2 \theta}(t) q_{2 \psi}(t)\right)^{T} \in \mathbb{R}^{3}, u_{q 1}(t)=\left(x_{1}(t) u(t)\right)^{T} \in$
$\mathbb{R}^{6 \times 1}, u_{q 2}(t)=\left(x_{1}(t) q_{1}(t)\right)^{T} \in \mathbb{R}^{6 \times 1}, \lambda=\operatorname{diag}\left(-g_{\phi}{ }^{2}\right.$, $\left.-g_{\theta}{ }^{2},-g_{\psi}^{2}\right)$

$$
\begin{aligned}
A_{q 1} & =\left(\begin{array}{ccc}
-g_{\phi} & 0 & 0 \\
0 & -g_{\theta} & 0 \\
0 & 0 & -g_{\psi}
\end{array}\right) \in \mathbb{R}^{3 \times 3}, \\
B_{q 1} & =\left(\begin{array}{cccccc}
-g_{\phi}{ }^{2} & 0 & 0 & V_{\phi}^{N} & 0 & 0 \\
0 & -g_{\theta}{ }^{2} & 0 & 0 & V_{\theta}^{N} & 0 \\
0 & 0 & -g_{\psi}{ }^{2} & 0 & 0 & V_{\psi}^{N}
\end{array}\right) \\
& \left.\in \mathbb{R}^{3 \times 6}, \begin{array}{cccccc}
-g_{\phi} & 0 & 0 \\
0 & -g_{\theta} & 0 \\
0 & 0 & -g_{\psi}
\end{array}\right) \in \mathbb{R}^{3 \times 3}, \\
A_{q 2} & =\left(\begin{array}{cccccc}
2 g_{\phi} & 0 & 0 & 1 & 0 & 0 \\
0 & 2 g_{\theta} & 0 & 0 & 1 & 0 \\
0 & 0 & 2 g_{\psi} & 0 & 0 & 1
\end{array}\right) \in \mathbb{R}^{3 \times 6} .
\end{aligned}
$$

Remark 7. As can be seen, the designed control input $u(t)$ in (18) is achieved by the combination of the control input computed by the optimal LQR controller in (22) and the compensation input in (33). The proposed robust control design is linear time-invariant controller and can easily be implemented in practical applications.

Remark 8. For real-time implementation in an embedded system, (31) and (32) can be realised in discrete form by using several methods such as fourth-order Runge-Kutta, Euler, and Adams-Bashforth methods [32].

\section{Robust Properties Analysis}

Theorem 9. If Assumptions 1-4 are met, there is a sufficiently large $g_{\phi}, g_{\theta}$, and $g_{\psi}$, a finite-positive constant $T^{*}$ for any bounded initial state $x(0)$, and a specified positive constant $\varepsilon$. Therefore, the state $x(t)$ is bounded, satisfying $|x(t)| \leq \varepsilon$, $\forall t \geq T^{*}$.

Proof. From (25) and (30),

$$
\|x\|_{\infty} \leq \xi_{x(0)}+\delta_{G}\|\Delta\|_{\infty},
$$

where $\delta_{G}=\left\|\left(s I_{9 \times 9}-A_{H}\right)^{-1} B\left(I_{3 \times 3}-G(s)\right)\right\|_{1}, I_{n \times n}$ is an $n \times n$ unit matrix, $\xi_{x(0)}=\max _{k} \sup _{t \geq 0}\left|c_{k}^{T} x^{A_{H}^{t}} x(0)\right|$, and $c_{k}$ is a $9 \times 1$ vector with zeros except one on the $k$ th row. If positive constants $g_{\phi}, g_{\theta}$, and $g_{\psi}$ are sufficiently large, then $\delta_{G}$ can be made as small as desired because the gains of the low-pass filter $G(s)$ are gotten closer to $I_{3 \times 3}$.

From (15), there exist positive constants $\zeta_{\Delta x 0}, \zeta_{\Delta x 1}$, and $\zeta_{\Delta x 2}$ such that

$$
\|\Delta\|_{\infty} \leq \zeta_{\Delta x 1}\|x\|_{\infty}+\zeta_{\Delta x 2}\|x\|_{\infty}^{2}+\rho\|u\|_{\infty}+\zeta_{\Delta x 0}
$$




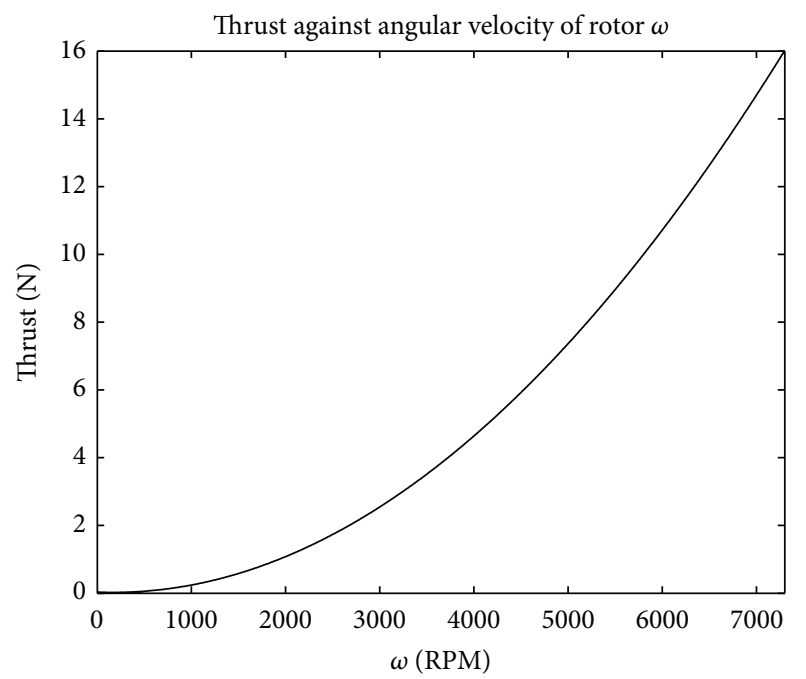

(a)

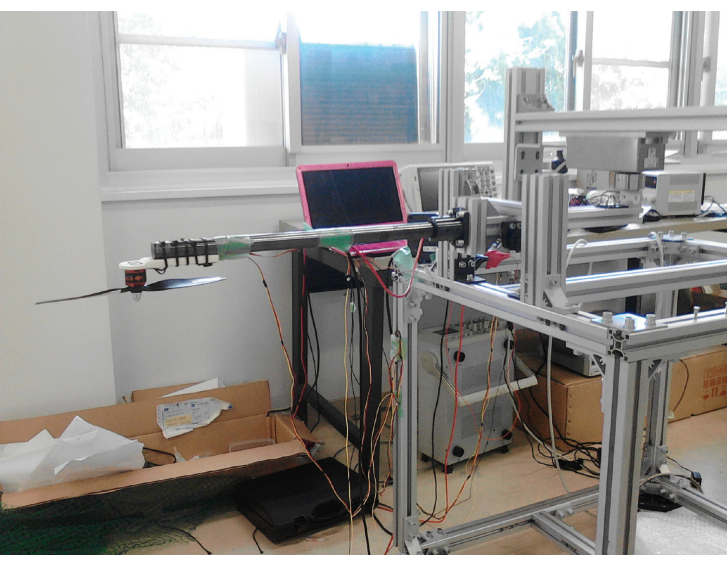

(b)

FIgURE 4: (a) The result and (b) experimental setup of static thrust test.

where $\rho=\max \left\{\rho_{\phi}, \rho_{\theta}, \rho_{\psi}\right\}<1$. Based on (18), (22), and (30), one has

$$
\|u\|_{\infty} \leq\|K\|_{1}\|x\|_{\infty}+\|\Delta\|_{\infty} .
$$

Substituting (37) into (36), it can be obtained that

$$
\|\Delta\|_{\infty} \leq \xi_{\Delta x 1}\|x\|_{\infty}+\xi_{\Delta x 2}\|x\|_{\infty}^{2}+\xi_{\Delta x 0},
$$

where $\xi_{\Delta x 1}=\left(\zeta_{\Delta x 1}+\rho\|K\|_{1}\right) /(1-\rho), \xi_{\Delta x 2}=\zeta_{\Delta x 2} /(1-\rho)$, and $\xi_{\Delta x 0}=\zeta_{\Delta x 0} /(1-\rho)$.

If $\delta_{G}$ is sufficiently small and satisfy

$$
\left(\xi_{\Delta x 1}+\xi_{\Delta x 2}\|x\|_{\infty}\right)\left(\sqrt{\delta_{G}}+\delta_{G}\right) \leq 1,
$$

therefore, from (35) and (38), it can be obtained that

$$
\|\Delta\|_{\infty} \leq \frac{\xi_{\Delta \delta}}{\sqrt{\delta_{G}}}
$$

where $\xi_{\Delta \delta}$ is a positive constant with $\xi_{\Delta \delta} \geq \xi_{x(0)}+\left(\sqrt{\delta_{G}}+\right.$ $\left.\delta_{G}\right) \xi_{\Delta x 0}$. From (25), (30), and (40), one has

$$
\max _{k}\left|x_{k}(t)\right| \leq \max _{k}\left|c_{k}^{T} x^{A_{H} t} x(0)\right|+\sqrt{\delta_{G}} \xi_{\Delta \delta} .
$$

Substituting (40) into (35) can be expressed as

$$
\|x\|_{\infty} \leq \xi_{x(0)}+\sqrt{\delta_{G}} \xi_{\Delta \delta} .
$$

Therefore, by choosing sufficiently large positive constants $g_{\phi}, g_{\theta}$, and $g_{\psi}$, the state $x(t)$ is bounded and satisfies $|x(t)| \leq \varepsilon, \forall t \geq T^{*}$.

From (39), the attractive region of $x(t)$ can be computed as

$$
\left\{x(t):\|x\|_{\infty} \leq \frac{\xi_{\mathrm{ar}}}{\sqrt{\delta_{G}}}\right\}
$$

where $\xi_{\text {ar }}>0$ satisfy

$$
\xi_{\mathrm{ar}}>\left(\left(1+\sqrt{\delta_{G}}\right) \xi_{\Delta x 2}\right)^{-1}-\left(\frac{\xi_{\Delta x 1} \sqrt{\delta_{G}}}{\xi_{\Delta x 2}}\right) .
$$

If $x(t)$ starts from the attractive region and the following inequality is met

$$
\xi_{x(0)}+\sqrt{\delta_{G}} \xi_{\Delta \delta} \leq \frac{\xi_{\mathrm{ar}}}{\sqrt{\delta_{G}}}
$$

$x(t)$ can remain in the attractive region. The inequality (45) can hold if $\delta_{G}$ is sufficiently small. If the initial state is

$$
\|x(0)\|_{\infty} \leq \frac{\xi_{\mathrm{ar}}}{\sqrt{\delta_{G}}},
$$

then the inequality (39) can hold.

Remark 10. Actually, the values of uncertainties bounds are difficult to obtain in real situations. However, the disturbance observer parameters $g_{\phi}, g_{\theta}$, and $g_{\psi}$ can be determined by an online tuning procedure: set $g_{\phi}$ from a small positive value (e.g., 0.5) and increase it in small step until satisfactory tracking performance is achieved. The similar tuning procedure is applied for $g_{\theta}$ and $g_{\psi}$.

\section{Simulation Results}

Several simulation tests were carried out to evaluate the attitude tracking performances of the proposed robust optimal attitude control design in the presence of the total disturbance which includes parametric uncertainties, external timevarying disturbances, nonlinear dynamics, and coupling. The nominal parameters of hexarotor model are based on the actual hexarotor research platform as presented in Table 1. The nominal values of thrust coefficient $b^{N}$ and torque constant $k^{N}$ were obtained by the static thrust test. Figure 4 
TABLE 1: Hexarotor nominal parameters.

\begin{tabular}{lcc}
\hline Nominal parameter & Nominal value & Unit \\
\hline$m^{N}$ & 1.52 & $\mathrm{~kg}$ \\
$g^{N}$ & 9.81 & $\mathrm{~m} / \mathrm{s}^{2}$ \\
$d^{N}$ & 0.275 & $\mathrm{~m}$ \\
$b^{N}$ & $2.8625 \times 10^{-7}$ & \\
$k^{N}$ & $4.4212 \times 10^{-10}$ & \\
$J_{x x}{ }^{N}$ & 0.0358 & $\mathrm{kgm}^{2}$ \\
$J_{y y}{ }^{N}$ & 0.0357 & $\mathrm{kgm}^{2}$ \\
$J_{z z}{ }^{N}$ & 0.0728 & $\mathrm{kgm}^{2}$ \\
\hline
\end{tabular}

shows the result and experimental setup of the static thrust test. The details of the static thrust test for rotor identification can be found in $[31,33]$. Based on Table 1 , the parameter matrix $V^{N}=\operatorname{diag}\left(d^{N} b^{N} / J_{x x}{ }^{N}, d^{N} b^{N} / J_{y y}{ }^{N}, k^{N} / J_{z z}{ }^{N}\right)$. Simulation tests were conducted in MATLAB and Simulink environments. The dynamics model of the hexarotor used in this section consists of nonlinearities and coupling. In this section, the hexarotor is assumed to be attached on the spherical joint for attitude test only without translational motion (e.g., see [19]). The control input $u_{T}$ is assumed to be $\sqrt{m^{N} g^{N} / 6 b^{N}}$ in order to produce the thrust equal to the weight of the hexarotor in hovering conditions [34]. In practice, $u_{T}$ will be produced by altitude controller.

Three different cases are presented in this section. Firstly, the optimal LQR attitude controller is designed and evaluated without the effects of parametric uncertainties and external time-varying disturbances. After that, the total disturbance is considered and the optimal LQR attitude controller design is evaluated under the effects of the total disturbance. Finally, the proposed robust optimal attitude controller is evaluated under the effects of the total disturbance and its performances are compared with the optimal LQR controller. For each case, two different missions have been considered which are hovering and aggressive missions. In hovering mission, the hexarotor has to stabilize and the desired attitude is $r(t)=\left(\begin{array}{lll}0^{\circ} & 0^{\circ} & 0^{\circ}\end{array}\right)^{T}$. In aggressive mission, the large attitude reference is considered as

$$
r(s)=\left(\begin{array}{c}
\frac{p_{\phi}(s)}{(s+1)^{2}} \\
\frac{p_{\theta}(s)}{(s+1)^{2}} \\
\frac{p_{\psi}(s)}{(s+1)^{2}}
\end{array}\right),
$$

where $p_{\phi}(s), p_{\theta}(s)$, and $p_{\psi}(s)$ are periodic square waveforms.

5.1. Case 1: Optimal LQR Attitude Controller without the Effects of Parametric Uncertainties and External TimeVarying Disturbances. The optimal LQR attitude controller is designed to achieve good attitude tracking performances for the nominal system. In this case, the total disturbance is small because it is only influenced by nonlinearities and coupling effects. Thus, the effects of the total disturbance can
TABLE 2: Controller parameters.

\begin{tabular}{lc}
\hline Optimal LQR controller parameter & $\begin{array}{c}\text { Disturbance } \\
\text { observer } \\
\text { parameter }\end{array}$ \\
\hline$r=\operatorname{diag}(1000,1000,1000)$ & $g_{\phi}=1$ \\
$Q=\operatorname{diag}(700,700,700,1,1,1,0.002,0.002,15)$ & $g_{\theta}=2$ \\
& $g_{\psi}=5$ \\
\hline
\end{tabular}

be ignored. However, the initial attitude in hovering mission is $\eta(0)=\left(\begin{array}{lll}-1^{\circ} & 1^{\circ} & 0^{\circ}\end{array}\right)^{T}$ in order to show the transients when reaching the hover. The optimal LQR parameters $r$ and $Q$ used in this case are presented in Table 2. Figures 5 and 7 show the attitude tracking responses of the optimal LQR controller for hovering and aggressive missions, respectively. The attitude control inputs for hovering and aggressive missions are presented in Figures 6 and 8, respectively. Based on the attitude tracking responses, the optimal LQR controller achieved excellent attitude tracking performances for both missions without the effects of parametric uncertainties and external time-varying disturbances. It is proven that the influence of the nonlinear dynamics and coupling could be restrained based on the accurate dynamics model of hexarotor. Based on LQR control theory [35], the closed-loop dynamics are guaranteed to be asymptotically stable if the state $x$ is available for feedback, $\left[\begin{array}{ll}A & B\end{array}\right]$ is stabilizable, and $r=r^{T}>0$.

5.2. Case 2: Optimal LQR Attitude Controller under the Effects of the Total Disturbance. In this case, the effects of the total disturbance are considered which includes parametric uncertainties, external time-varying disturbances, nonlinear dynamics, and coupling. The uncertain part of parameter matrix $V^{\Delta}$ is assumed to be $90 \%$ of the nominal value $V^{N}$ which represents a large amount of parametric uncertainties and the external time-varying disturbance $w(t)$ is assumed to be

$$
w(t)=\left(\begin{array}{c}
70 \sin 1.2 t \\
60 \sin 1.2 t \\
5000 \sin 0.5 t
\end{array}\right)
$$

Then, the performances of the designed optimal LQR attitude controller from previous case are evaluated under the effects of the total disturbance. The initial attitude for hovering mission in this case is $\eta(0)=\left(\begin{array}{lll}0^{\circ} & 0^{\circ} & 0^{\circ}\end{array}\right)^{T}$. Based on Figures 9 and 11, the optimal LQR controller is unable to sufficiently track the reference signals for both hovering and aggressive missions. Thus, the attitude tracking errors are increased. The attitude control inputs for hovering and aggressive missions are presented in Figures 10 and 12, respectively. Based on results, the optimal LQR controller is not robust against the total disturbance due to imperfections of the dynamics model. Actually, the model-based control approach like the optimal LQR controller needs an accurate model in order to achieve the desired tracking performances. 

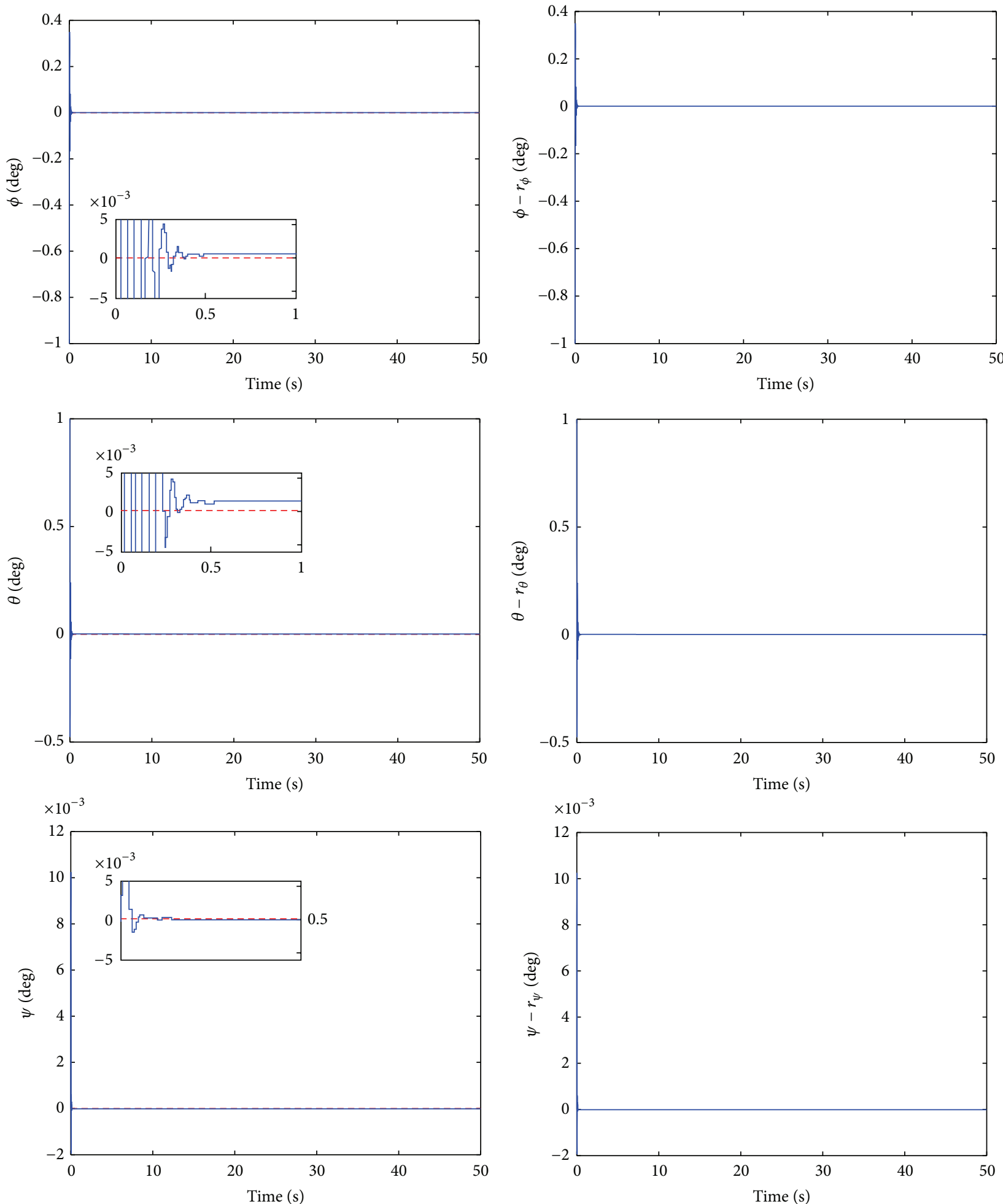

(a)

(b)

FIGURE 5: Case 1: hovering mission. (a) and (b) show the attitude tracking responses and the attitude tracking errors, respectively, of the optimal LQR controller. The red dashed line in (a) represents the attitude reference signal. 

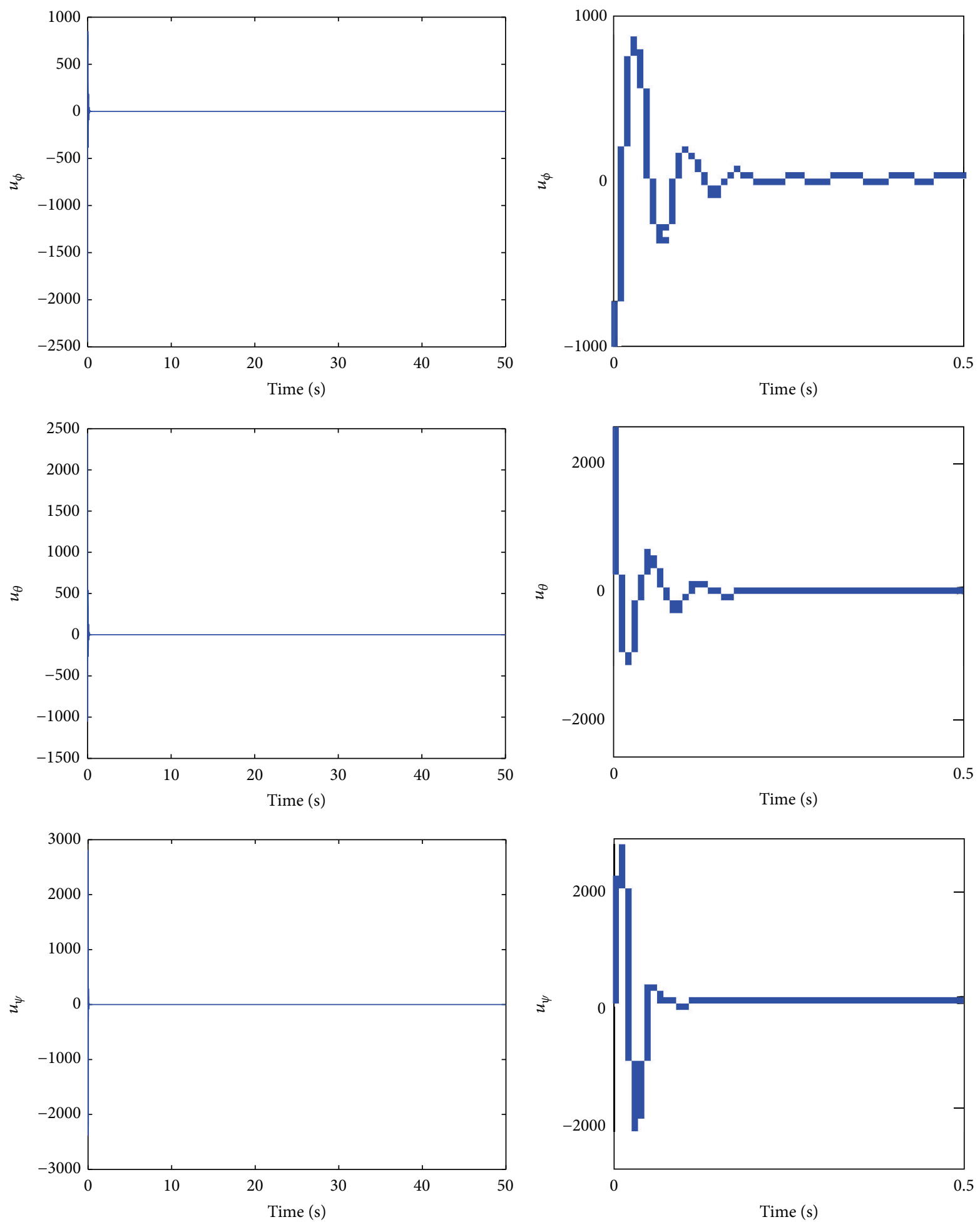

(a)

(b)

FIGURE 6: Case 1: hovering mission. The attitude control inputs of the optimal LQR controller.

However, in reality, an exact mathematical model of hexarotor cannot be obtained especially in outdoor flying environments because the model is only an approximation of the real system.
5.3. Case 3: Proposed Robust Optimal Attitude Controller under the Effects of the Total Disturbance. In this case, the uncertain part of parameter matrix $V^{\Delta}$ and the external time-varying disturbance $w(t)$ are similar to those in Case 2. 

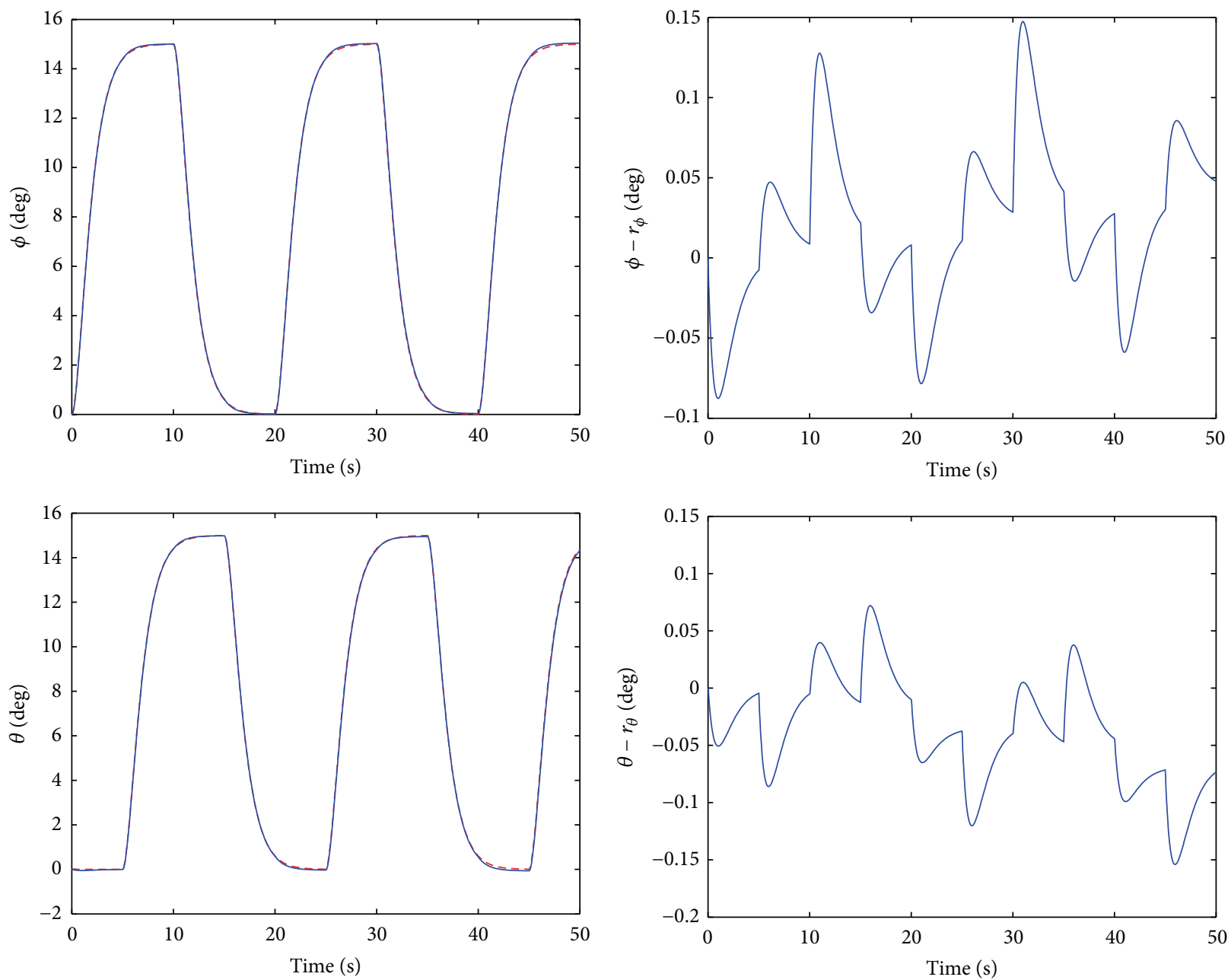

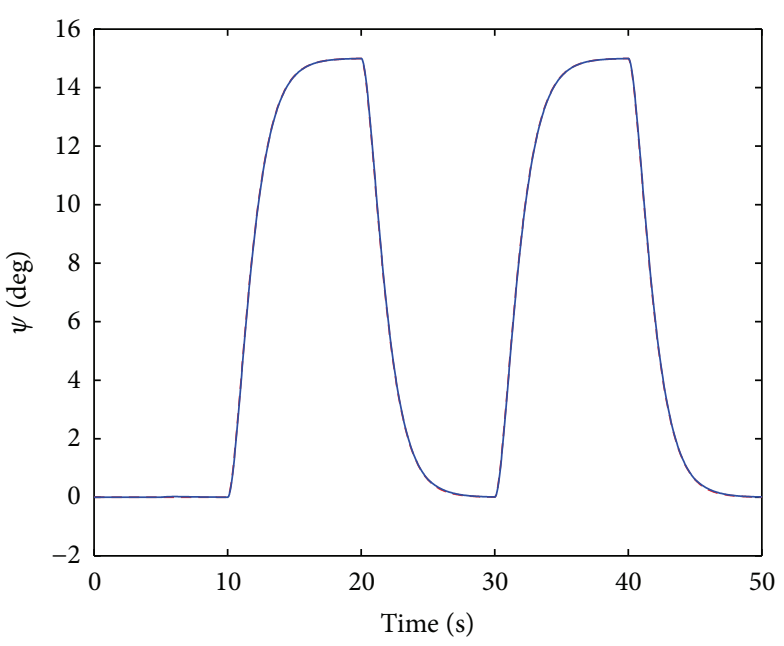

(a)

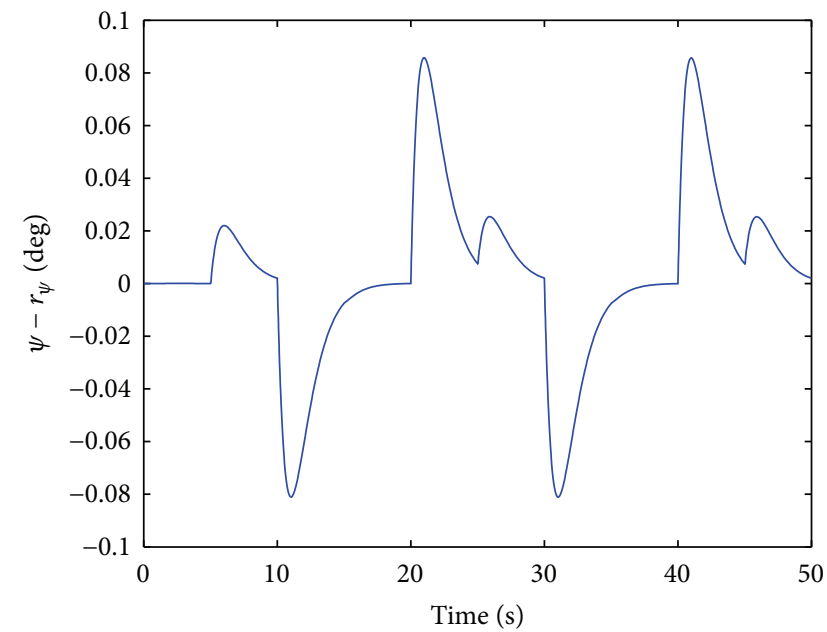

(b)

FIGURE 7: Case 1: aggressive mission. (a) and (b) show the attitude tracking responses and the attitude tracking errors, respectively, of the optimal LQR controller. The red dashed line in (a) represents the attitude reference signal. 

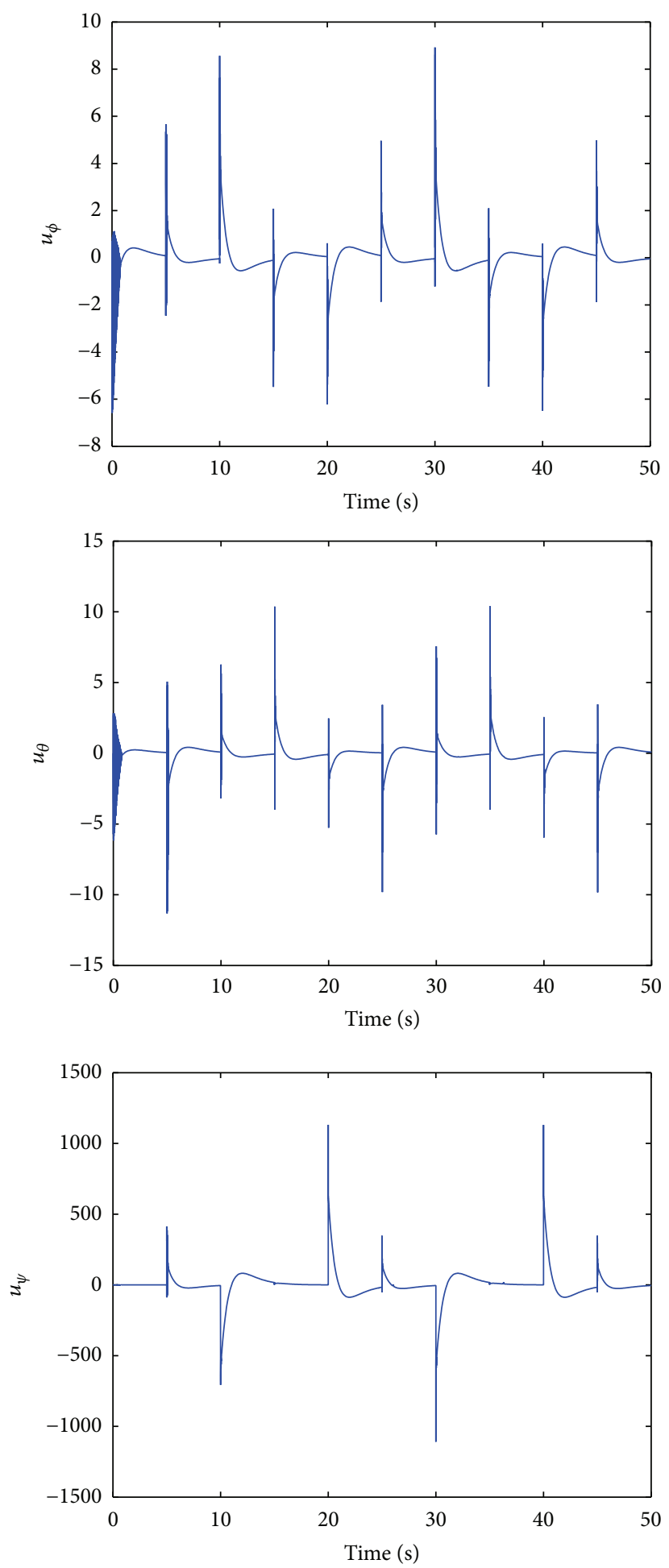

FIgURE 8: Case 1: aggressive mission. The attitude control inputs of the optimal LQR controller.

The disturbance observer was integrated into the existing closed-loop system to estimate the total disturbance. The disturbance observer parameters are presented in Table 2. Please refer to Remark 10 in Section 4 for online tuning procedure of disturbance observer parameters. It should be highlighted that the proposed robust optimal controller is a combination of the optimal LQR controller from previous case and the disturbance observer.

First, the proposed robust optimal controller is evaluated in hovering mission. The initial attitude in hovering mission 

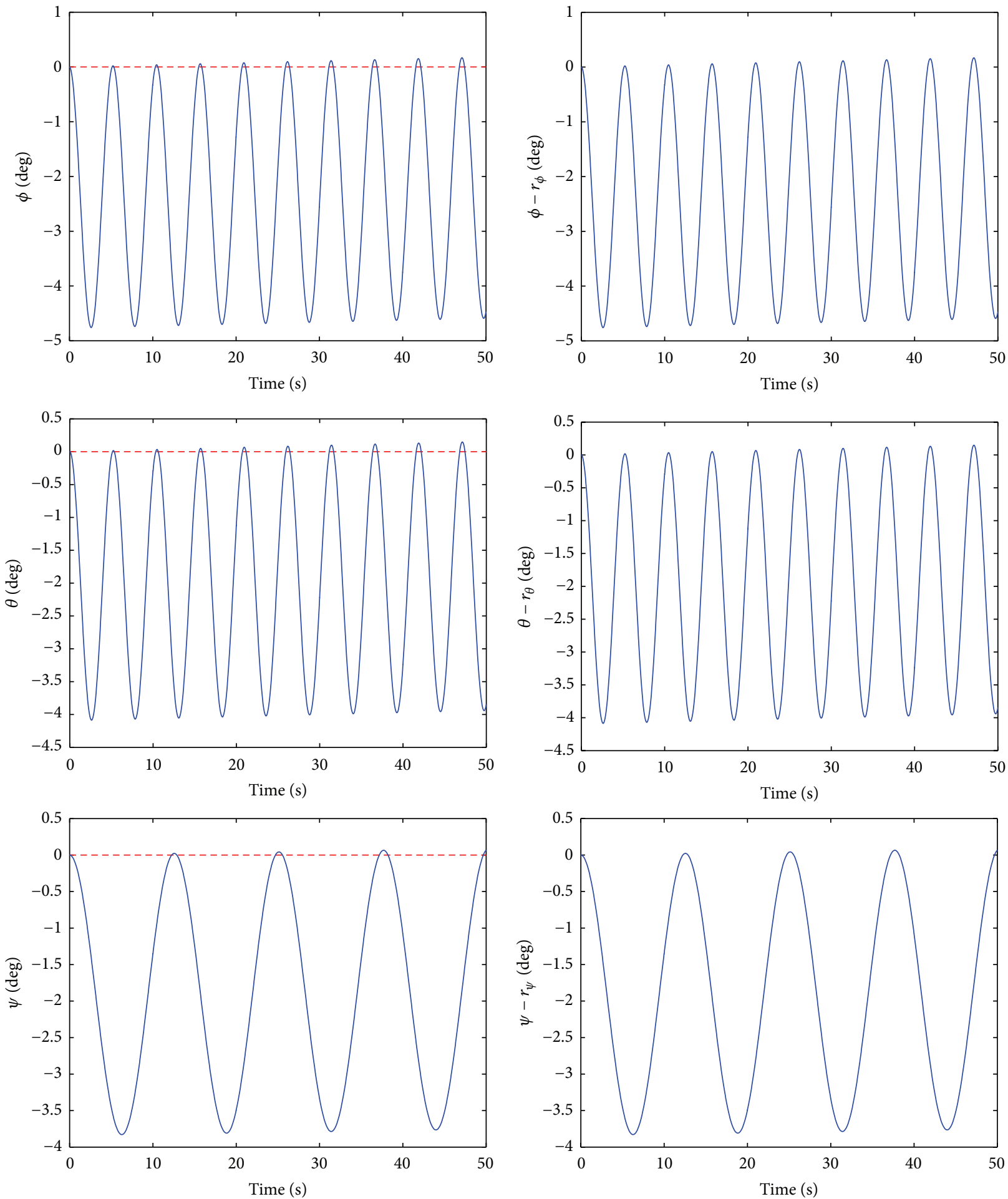

(a)

(b)

FIGURE 9: Case 2: hovering mission. (a) and (b) show the attitude tracking responses and the attitude tracking errors, respectively, of the optimal LQR controller. The red dashed line in (a) represents the attitude reference signal. 

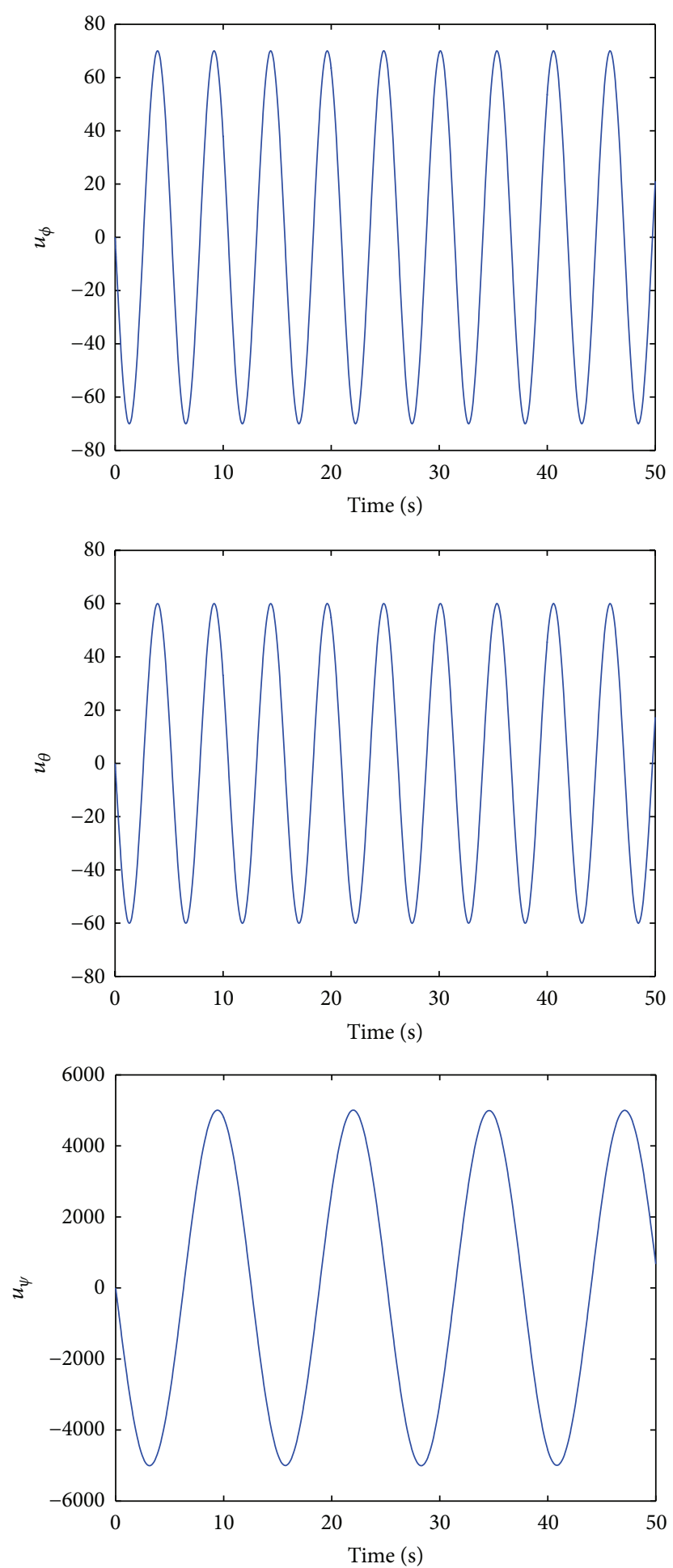

FIGURE 10: Case 2: hovering mission. The attitude control inputs of the optimal LQR controller.

is $\eta(0)=\left(\begin{array}{lll}0^{\circ} & 0^{\circ} & 0^{\circ}\end{array}\right)^{T}$. Figures 13 and 14 show the attitude tracking responses and the attitude control inputs of the proposed robust optimal controller in hovering mission, respectively. As can be seen, the proposed robust control scheme achieved good steady-state tracking performances in the presence of the total disturbance. It is also proven that the attitude tracking errors are ultimately bounded in $\left(2 \times 10^{-4}\right)^{\circ},\left(5 \times 10^{-5}\right)^{\circ}$, and $\left(2 \times 10^{-6}\right)^{\circ}$ for roll, pitch, and yaw angles, respectively. The total disturbance is successfully estimated by disturbance observer as presented in Figure 15. As can be seen, the estimated total disturbance $\widehat{\widehat{\Delta}}(t)=$ $\left(\widehat{\Delta}_{\phi}(t) \widehat{\Delta}_{\theta}(t) \widehat{\Delta}_{\psi}(t)\right)^{T}$ is almost similar to the external 

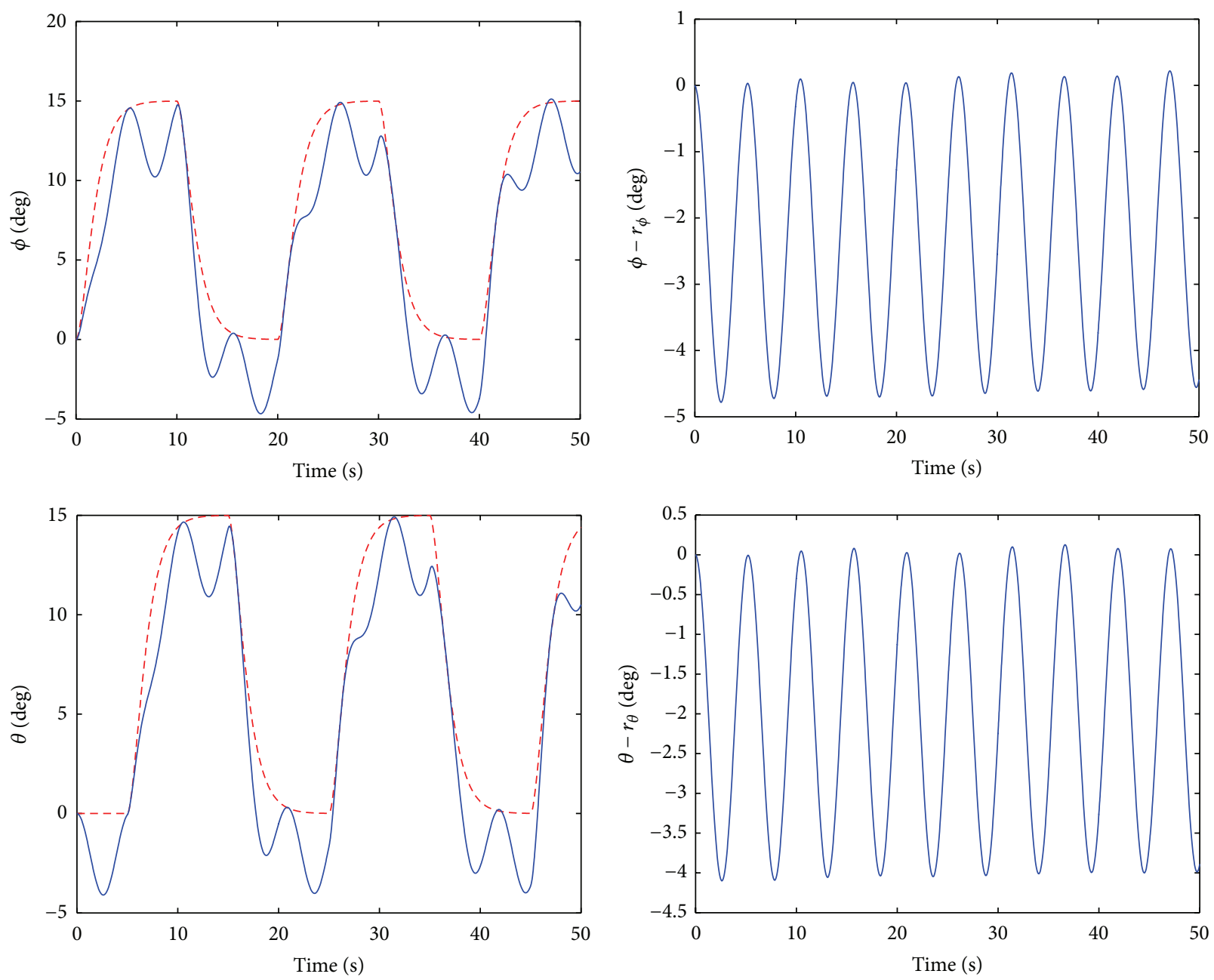

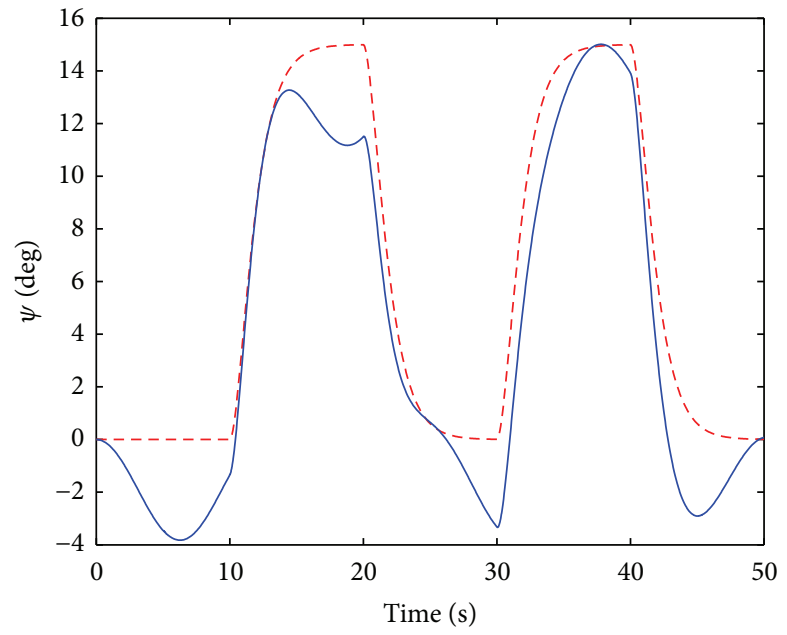

(a)

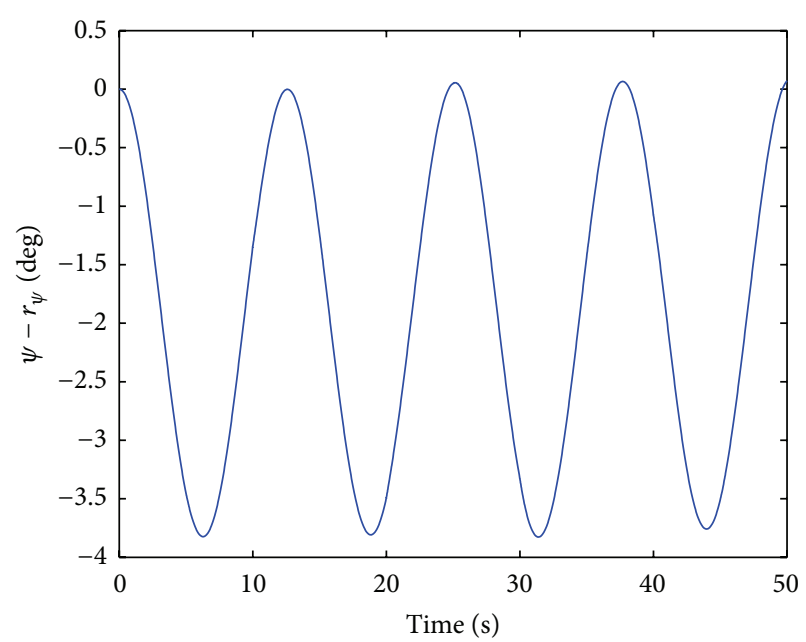

(b)

FIGURE 11: Case 2: aggressive mission. (a) and (b) show the attitude tracking responses and the attitude tracking errors, respectively, of the optimal LQR controller. The red dashed line in (a) represents the attitude reference signal. 

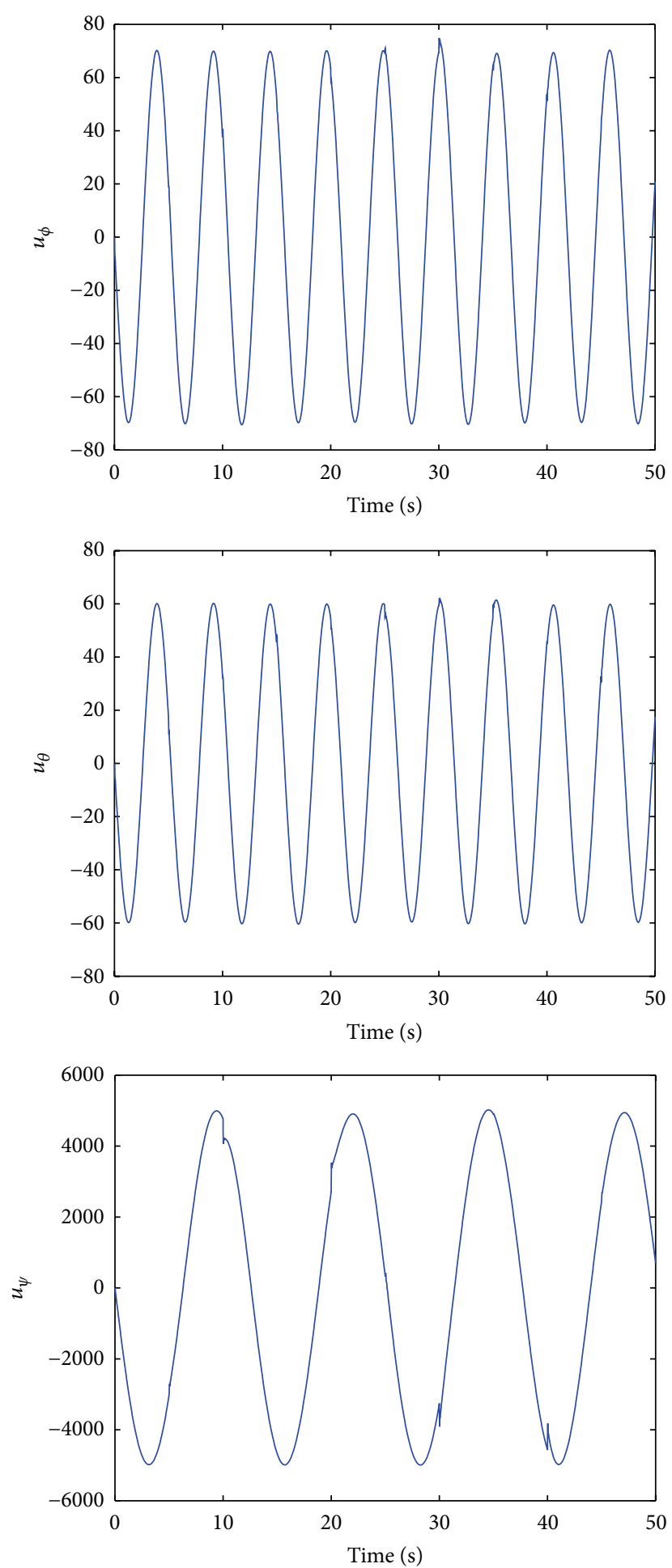

FIGURE 12: Case 2: aggressive mission. The attitude control inputs of the optimal LQR controller.

time-varying disturbance $w(t)=\left(w_{\phi}(t) w_{\theta}(t) w_{\psi}(t)\right)^{T}$ which is the major part of the total disturbance. In practical applications, the external time-varying disturbance would be the additional torque caused by wind disturbances.

Then, the proposed robust optimal controller is evaluated in aggressive mission. The attitude tracking responses and the attitude control inputs of the proposed robust optimal controller in aggressive mission are presented in Figures 16 and 17, respectively. As can be seen, the proposed attitude controller achieved excellent dynamical tracking performances. The estimated total disturbance against the external time-varying disturbance is illustrated in Figure 18. As can 

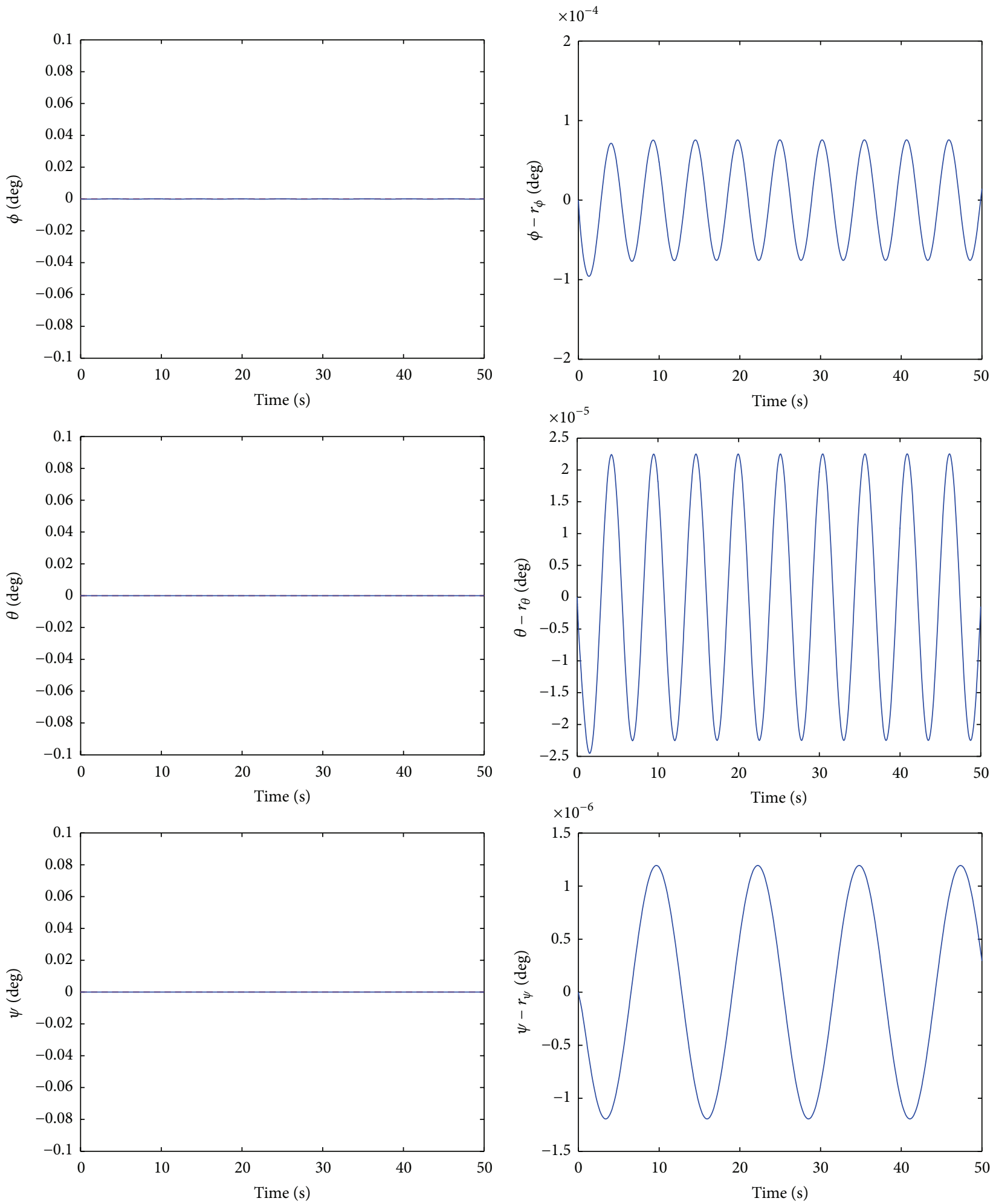

(a)

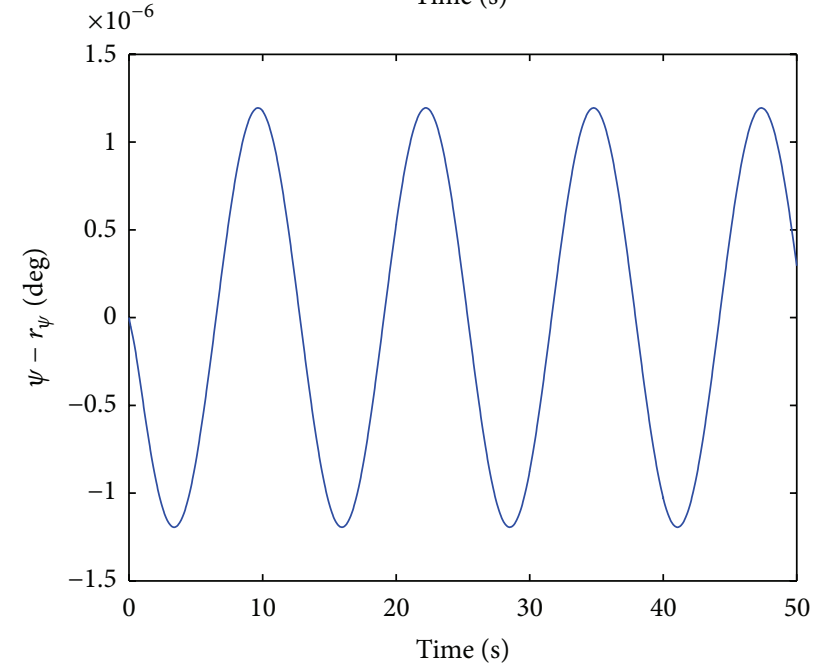

(b)

FIGURE 13: Case 3: hovering mission. (a) and (b) show the attitude tracking responses and the attitude tracking errors, respectively, of the proposed robust optimal controller. The red dashed line in (a) represents the attitude reference signal. 

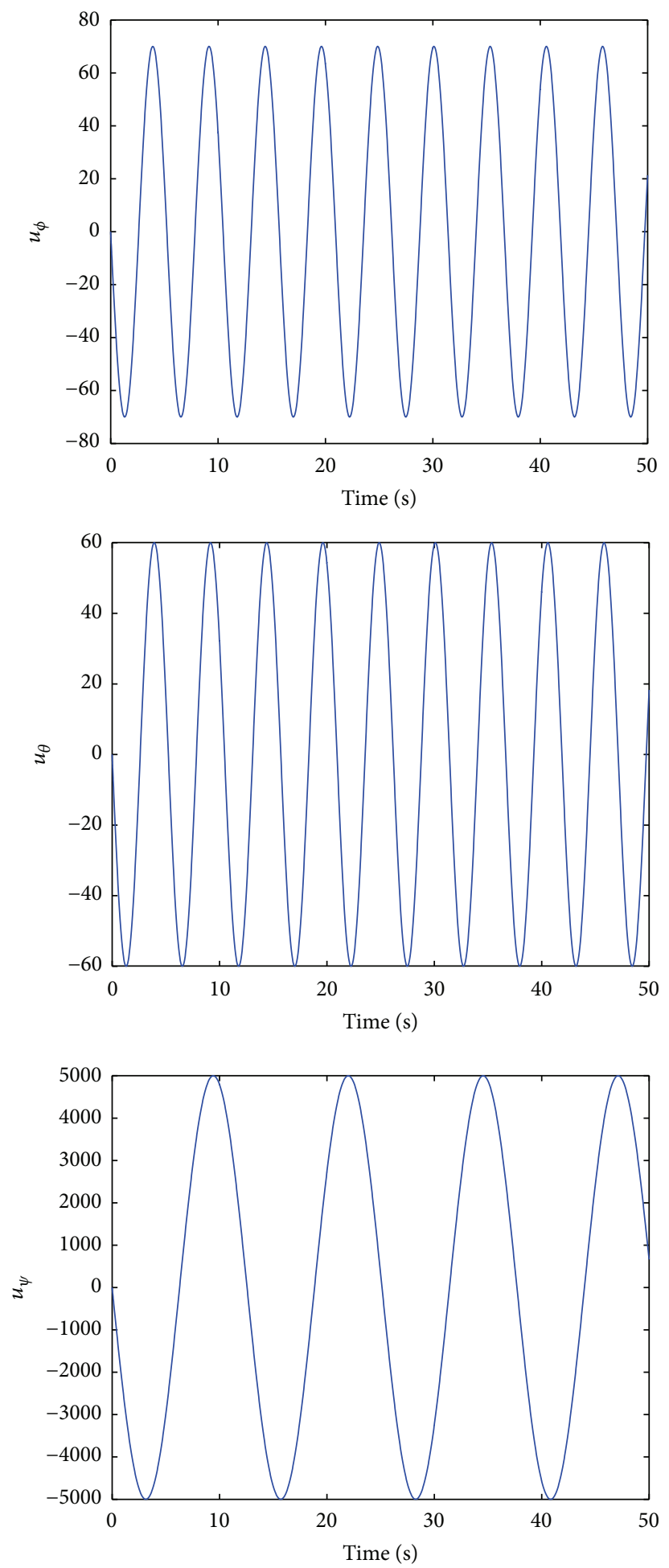

FIGURE 14: Case 3: hovering mission. The attitude control inputs of the proposed robust optimal controller.

be seen, there is a small glitch that occurred sometimes on the estimated total disturbance due to high coupling effects in aggressive mission. However, the total disturbance in the system has been successfully rejected. The attitude tracking errors are ultimately bounded in $\left(2 \times 10^{-4}\right)^{\circ},\left(5 \times 10^{-5}\right)^{\circ}$, and $\left(2 \times 10^{-6}\right)^{\circ}$ for roll, pitch, and yaw angles, respectively.
Compared to optimal LQR attitude controller as presented in Case 2 and previous model-based attitude controller design in [3-11], the proposed robust optimal controller is robust against multiple uncertainties and it does not require an accurate model in order to achieve the desired tracking performances. In addition, the proposed controller 

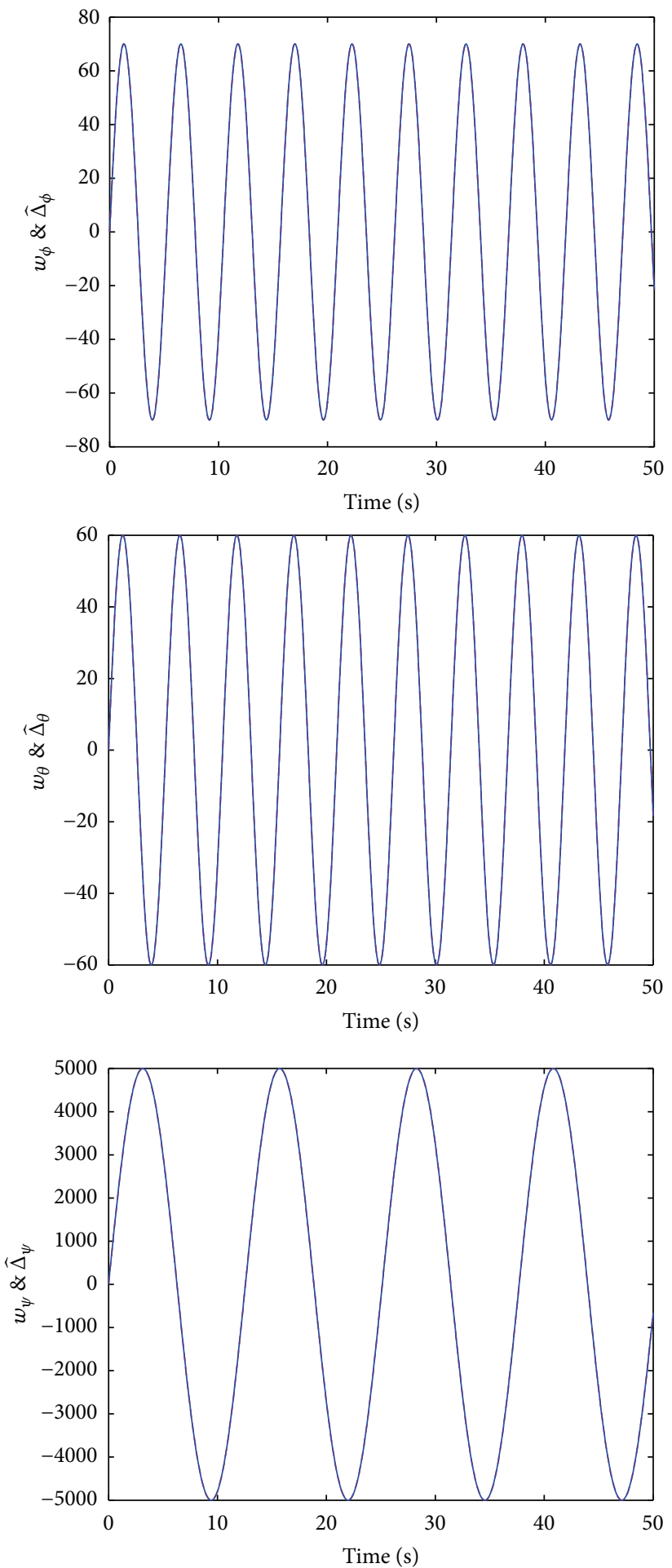

FIGURE 15: Case 3: hovering mission. The estimated total disturbance (the blue line) and the external time-varying disturbance (the red dashed line).

can improve the steady-state and dynamic attitude tracking performances by introducing the disturbance observer to estimate the total disturbance acting on the system. Compared to [16-20], the proposed method can specify the desired robust properties (e.g., the desired boundaries of attitude tracking errors). Based on the results in Case 3, the attitude tracking errors are ultimately bounded in specified boundaries for both missions $\left(\left(2 \times 10^{-4}\right)^{\circ},\left(5 \times 10^{-5}\right)^{\circ}\right.$, 

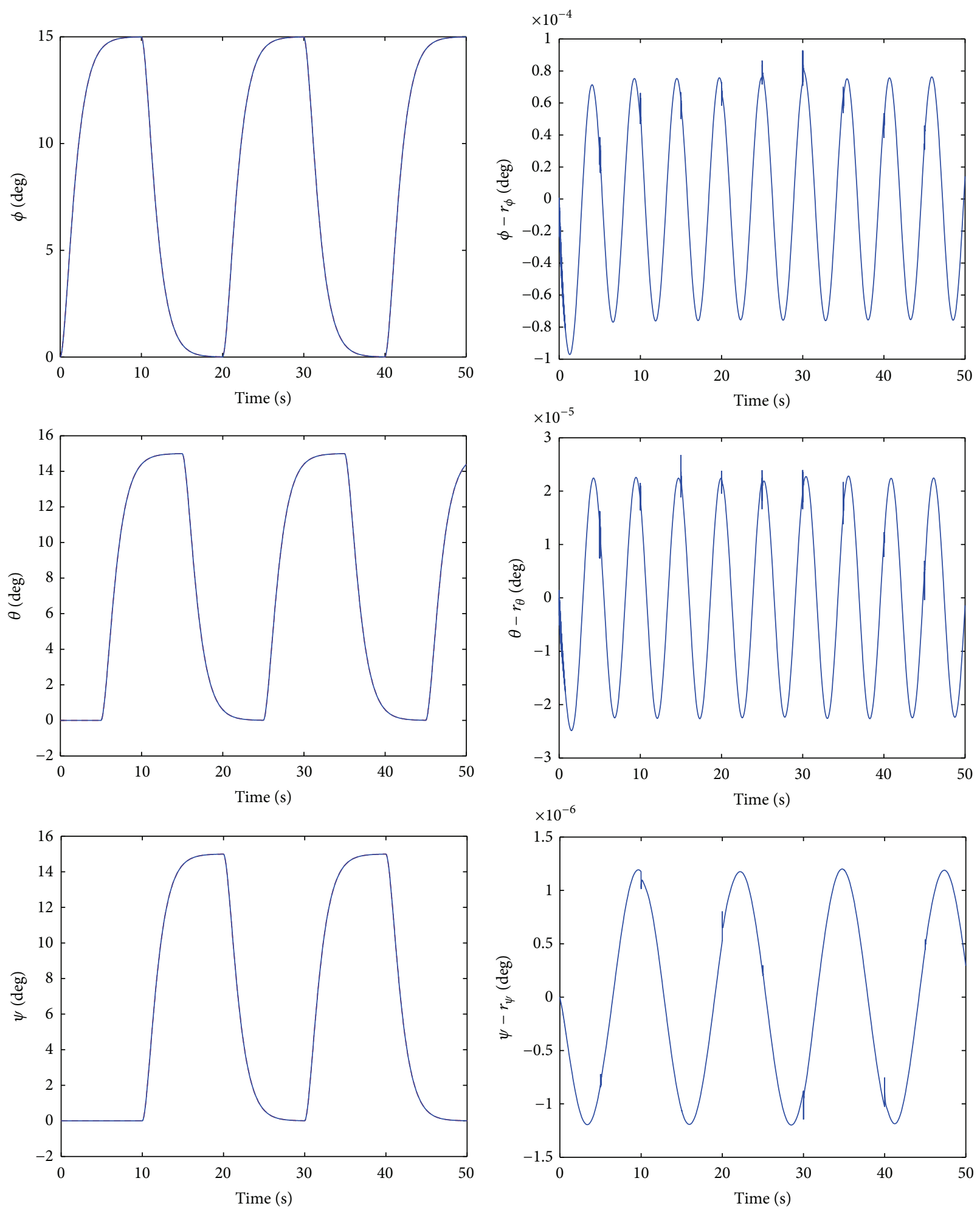

(a)

(b)

FIGURE 16: Case 3: aggressive mission. (a) and (b) show the attitude tracking responses and the attitude tracking errors, respectively, of the proposed robust optimal controller. The red dashed line in (a) represents reference signals. 

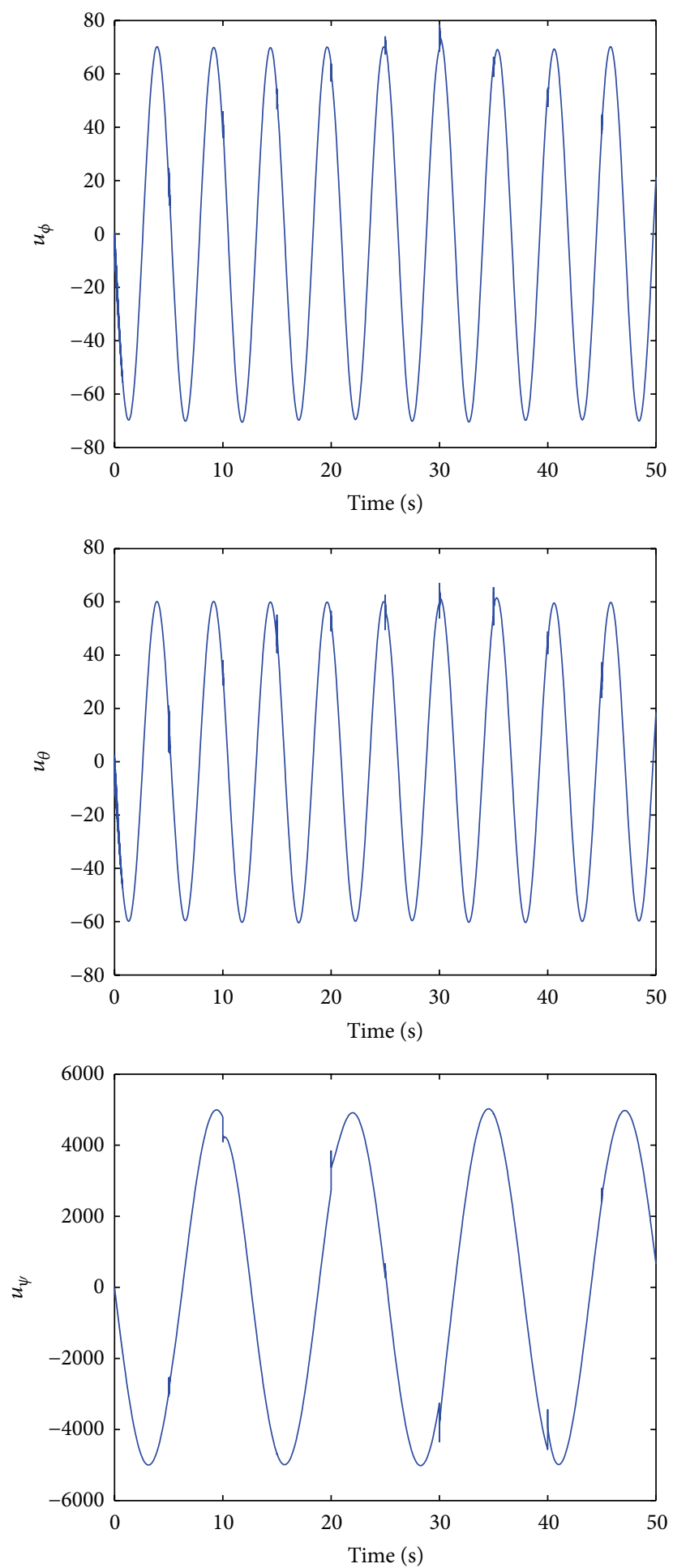

FIgURE 17: Case 3: aggressive mission. The attitude control inputs of the proposed robust optimal controller.

and $\left(2 \times 10^{-6}\right)^{\circ}$ for roll, pitch, and yaw angles, resp.).

\section{Conclusion}

This paper has proposed a robust optimal attitude control design for multiple-input multiple-output (MIMO) uncertain hexarotor micro aerial vehicles (MAVs) in the presence of parametric uncertainties, external time-varying disturbances, nonlinear dynamics, and coupling. The parametric uncertainties, external time-varying disturbances, nonlinear dynamics, and coupling have been treated as the total disturbance in the proposed design. The proposed controller is designed by the combination of the optimal linear-quadratic 

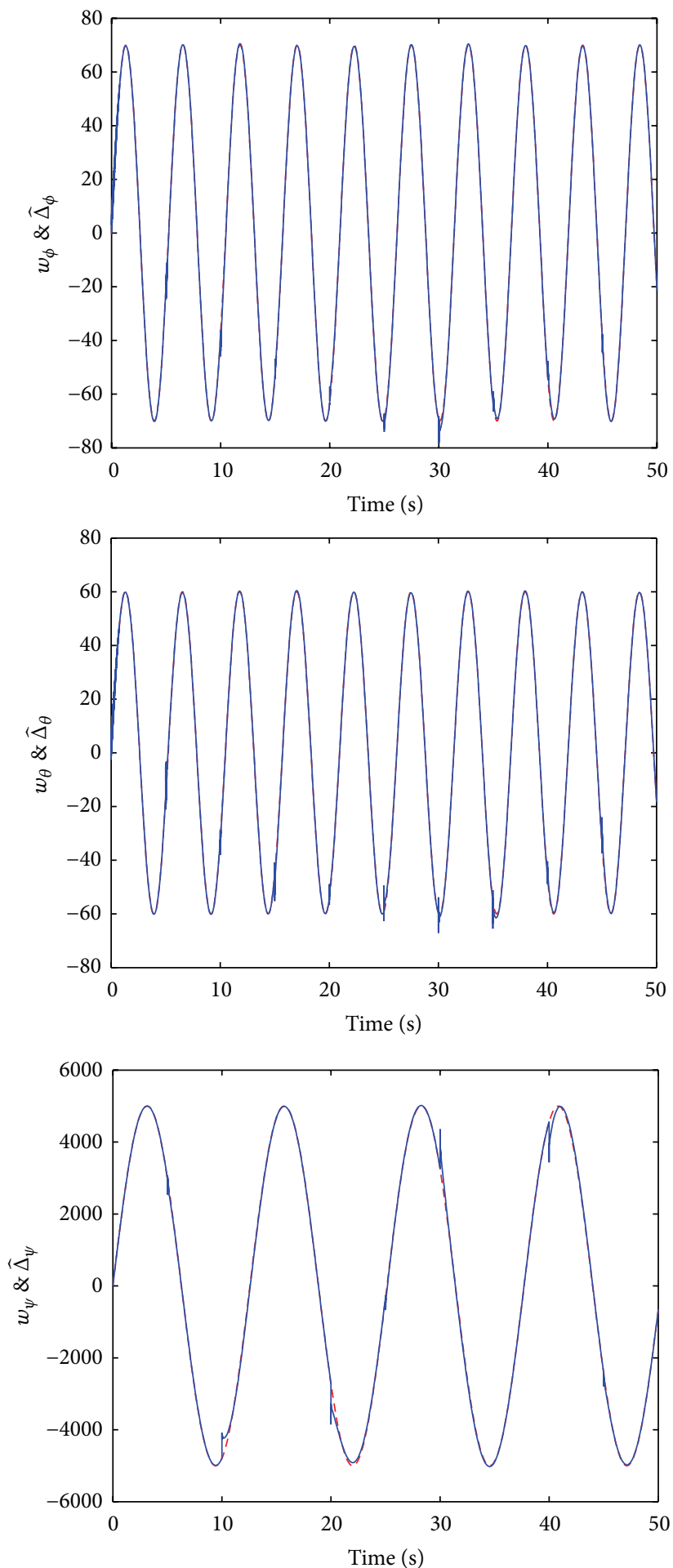

FIGURE 18: Case 3: aggressive mission. The estimated total disturbance (the blue line) and the external time-varying disturbance (the red dashed line).

regulator (LQR) controller and the disturbance observer. The total disturbance is estimated by the disturbance observer and is compensated from the system by the compensation input. Robust properties analysis proved that the state is ultimately bounded in specified boundaries. The simulation results demonstrated the robustness of the proposed controller for hovering and aggressive flight missions in the presence of the total disturbance, with excellent steady-state and dynamic attitude tracking performances. This research aims to integrate with the position controller for trajectory tracking. 


\section{Competing Interests}

The authors declare that they have no competing interests.

\section{Acknowledgments}

This research was mainly supported by Malaysia-Japan International Institute of Technology, Universiti Teknologi Malaysia (UTM), Autonomous Control Systems Laboratory Ltd. in Japan, Chiba University, and Robotic Systems Enterprise.

\section{References}

[1] P. Pounds, R. Mahony, and P. Corke, "Modelling and control of a large quadrotor robot," Control Engineering Practice, vol. 18, no. 7, pp. 691-699, 2010.

[2] S. Bouabdallah, A. Noth, and R. Siegwart, "PID vs LQ control techniques applied to an indoor micro quadrotor," in Proceedings of the IEEE/RSJ International Conference on Intelligent Robots and Systems, pp. 2451-2456, 2004.

[3] C. Aguilar-ibáñez, H. Sira-ramírez, M. S. Suárez-castañón, E. Martínez-navarro, and M. A. Moreno-Armendariz, "The trajectory tracking problem for an unmanned four-rotor system: flatness-based approach," International Journal of Control, vol. 85, no. 1, pp. 69-77, 2012.

[4] A. Das, K. Subbarao, and F. Lewis, "Dynamic inversion with zero-dynamics stabilisation for quadrotor control," IET Control Theory \& Applications, vol. 3, no. 3, pp. 303-314, 2009.

[5] S. Bertrand, N. Guénard, T. Hamel, H. Piet-Lahanier, and L. Eck, "A hierarchical controller for miniature VTOL UAVs: design and stability analysis using singular perturbation theory," Control Engineering Practice, vol. 19, no. 10, pp. 1099-1108, 2011.

[6] P. Castillo, A. Dzul, and R. Lozano, "Real-time stabilization and tracking of a four-rotor mini rotorcraft," IEEE Transactions on Control Systems Technology, vol. 12, no. 4, pp. 510-516, 2004.

[7] A. S. Sanca, P. J. Alsina, and J. F. Cerqueira, "Dynamic modeling with nonlinear inputs and backstepping control for a hexarotor micro-aerial vehicle," in Proceedings of the Latin American Robotics Symposium and Intelligent Robotics Meeting, pp. 36-42, 2010.

[8] K. Alexis, G. Nikolakopoulos, and A. Tzes, "Switching model predictive attitude control for a quadrotor helicopter subject to atmospheric disturbances," Control Engineering Practice, vol. 19, no. 10, pp. 1195-1207, 2011.

[9] A. Tayebi and S. McGilvray, "Attitude stabilization of a VTOL quadrotor aircraft," IEEE Transactions on Control Systems Technology, vol. 14, no. 3, pp. 562-571, 2006.

[10] R. Zawiski and M. Błachuta, "Modelling and optimal control system design for quadrotor platform - an extended approach," Bulletin of the Polish Academy of Sciences: Technical Sciences, vol. 62 , no. 3, pp. 535-550, 2014

[11] A. Honglei, L. Jie, W. Jian, W. Jianwen, and M. Hongxu, "Backstepping-based inverse optimal attitude control of quadrotor," International Journal of Advanced Robotic Systems, vol. 10, article 223, 2013.

[12] I.-H. Choi and H.-C. Bang, "Adaptive command filtered backstepping tracking controller design for quadrotor unmanned aerial vehicle," Proceedings of the Institution of Mechanical Engineers Part G: Journal of Aerospace Engineering, vol. 226, no. 5, pp. 483-497, 2012.
[13] Z. T. Dydek, A. M. Annaswamy, and E. Lavretsky, "Adaptive control of quadrotor UAVs: a design trade study with flight evaluations," IEEE Transactions on Control Systems Technology, vol. 21, no. 4, pp. 1400-1406, 2013.

[14] I. H. Choi and H. C. Bang, "Quadrotor-tracking controller design using adaptive dynamic feedback-linearization method," Proceedings of the Institution of Mechanical Engineers, Part G, vol. 228, no. 12, pp. 2329-2342, 2014.

[15] L. Sun and Z. Zuo, "Nonlinear adaptive trajectory tracking control for a quad-rotor with parametric uncertainty," Proceedings of the Institution of Mechanical Engineers, Part G, vol. 229, no. 9, pp. 1709-1721, 2015.

[16] S. Islam, P. X. Liu, and A. El Saddik, "Robust control of fourrotor unmanned aerial vehicle with disturbance uncertainty," IEEE Transactions on Industrial Electronics, vol. 62, no. 3, pp. 1563-1571, 2015.

[17] B. Zhao, B. Xian, Y. Zhang, and X. Zhang, "Nonlinear robust adaptive tracking control of a quadrotor UAV via immersion and invariance methodology," IEEE Transactions on Industrial Electronics, vol. 62, no. 5, pp. 2891-2902, 2015.

[18] M. A. Mohd Basri, A. R. Husain, and K. A. Danapalasingam, "Intelligent adaptive backstepping control for MIMO uncertain non-linear quadrotor helicopter systems," Transactions of the Institute of Measurement and Control, vol. 37, no. 3, pp. 345-361, 2015.

[19] T. Lee, "Robust adaptive attitude tracking on $\mathrm{SO}(3)$ with an application to a quadrotor UAV," IEEE Transactions on Control Systems Technology, vol. 21, no. 5, pp. 1924-1930, 2013.

[20] D. Cabecinhas, R. Cunha, and C. Silvestre, "A globally stabilizing path following controller for rotorcraft with wind disturbance rejection," IEEE Transactions on Control Systems Technology, vol. 23, no. 2, pp. 708-714, 2015.

[21] L. Besnard, Y. B. Shtessel, and B. Landrum, "Quadrotor vehicle control via sliding mode controller driven by sliding mode disturbance observer," Journal of the Franklin Institute, vol. 349, no. 2, pp. 658-684, 2012.

[22] N. J. S. Amlashi, M. Rezaei, H. Bolandi, and K. Sedigh, "Robust second order sliding mode control," International Journal of Control Theory and Computer Modelling, vol. 4, no. 1, pp. 9-25, 2014.

[23] E.-H. Zheng, J.-J. Xiong, and J.-L. Luo, "Second order sliding mode control for a quadrotor UAV," ISA Transactions, vol. 53, no. 4, pp. 1350-1356, 2014.

[24] C. Yang, Z. Yang, X. Huang, S. Li, and Q. Zhang, "Modeling and robust trajectory tracking control for a novel six-rotor unmanned aerial vehicle," Mathematical Problems in Engineering, vol. 2013, Article ID 673525, 13 pages, 2013.

[25] R. Zhang, Q. Quan, and K.-Y. Cai, "Attitude control of a quadrotor aircraft subject to a class of time-varying disturbances," IET Control Theory \& Applications, vol. 5, no. 9, pp. 1140-1146, 2011.

[26] K. Ohnishi and T. Murakami, "Advanced motion control in robotics," in Proceedings of the 15th Annual Conference of IEEE Industrial Electronics Society (IECON '89), vol. 2, pp. 356-359, Philadelphia, Pa, USA, November 1989.

[27] K. Ohnishi, M. Shibata, and T. Murakami, "Motion control for advanced mechatronics," IEEE/ASME Transactions on Mechatronics, vol. 1, no. 1, pp. 56-67, 1996.

[28] D. Derawi, N. D. Salim, H. Zamzuri, M. A. A. Rahman, and K. Nonami, "Robust attitude control design for a low-cost hexarotor micro aerial vehicle (MAV)," Transactions of the Institute of Measurement and Control, 2016. 
[29] W. Lin and H. Liu, "Robust hierarchical control for uncertain multivariable hexarotor systems," Mathematical Problems in Engineering, vol. 2015, Article ID 108481, 8 pages, 2015.

[30] H. Liu, D. Derawi, J. Kim, and Y. Zhong, "Robust optimal attitude control of hexarotor robotic vehicles," Nonlinear Dynamics, vol. 74, no. 4, pp. 1155-1168, 2013.

[31] R. Mahony, V. Kumar, and P. Corke, "Multirotor aerial vehicles: modeling, estimation, and control of quadrotor," IEEE Robotics \& Automation Magazine, vol. 19, no. 3, pp. 20-32, 2012.

[32] P. Benyon, "A review of numerical methods for digital simulation," SIMULATION, vol. 11, no. 5, pp. 219-238, 1968.

[33] M. Bangura and R. Mahony, "Nonlinear dynamic modeling for high performance control of a quadrotor," in Proceedings of the Australasian Conference on Robotics and Automation (ACRA '12), pp. 3-5, Australian Robotics and Automation Association, Wellington, Australia, 2012.

[34] P. Corke, Robotics, Vision and Control: Fundamental Algorithms in MATLAB, Springer Tracts in Advanced Robotics, Springer, Berlin, Germany, 2011.

[35] S. L. William, "Control system advanced methods," in The Control Systems Handbook, CRC Press, 2nd edition, 2010. 


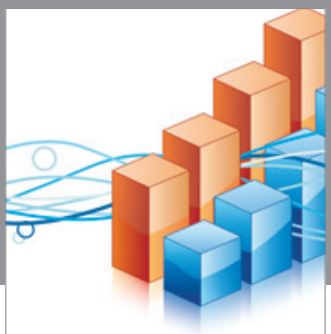

Advances in

Operations Research

vatem alat4

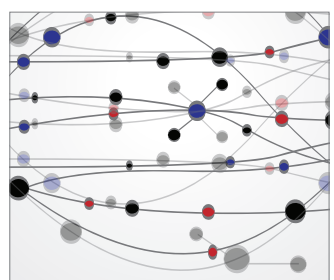

\section{The Scientific} World Journal
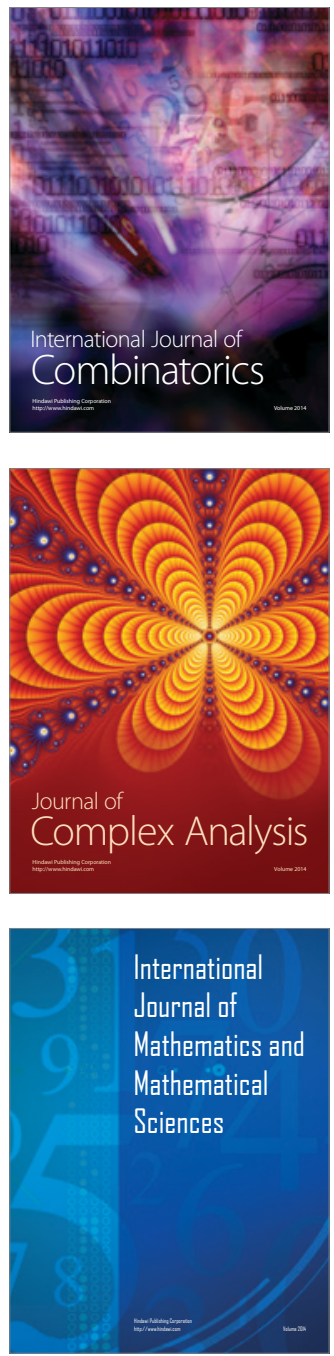
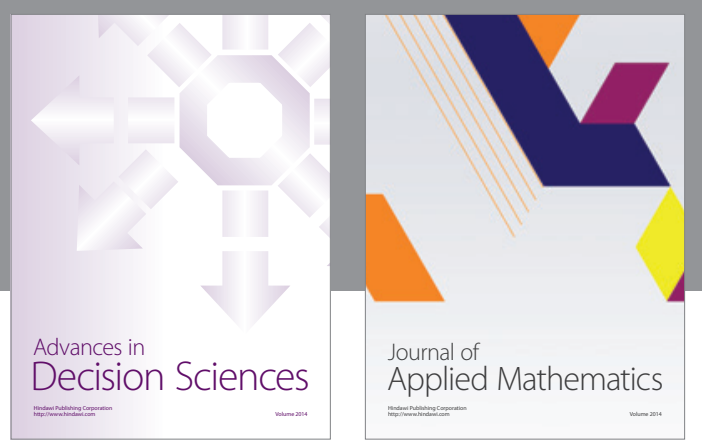

Algebra

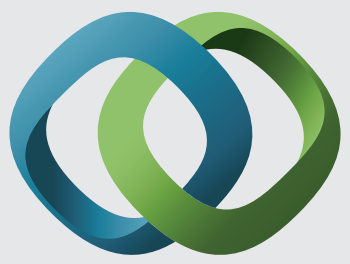

\section{Hindawi}

Submit your manuscripts at

http://www.hindawi.com
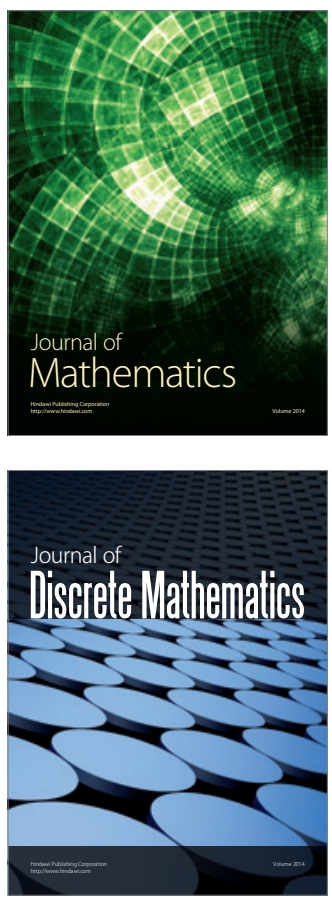

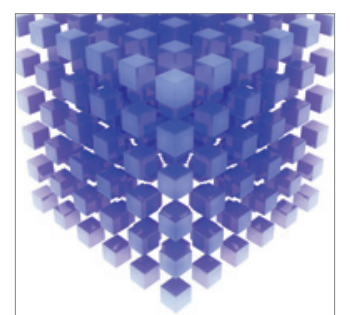

Mathematical Problems in Engineering
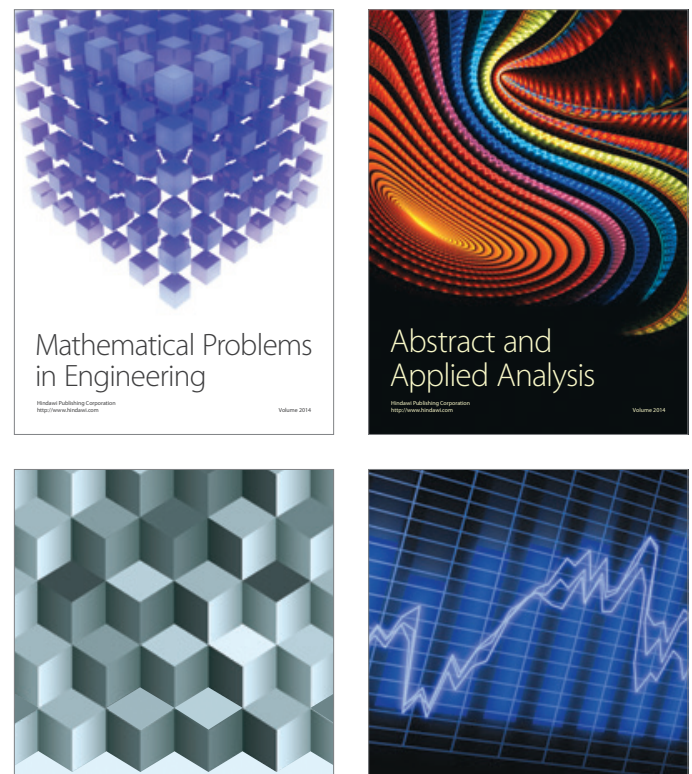

Journal of

Function Spaces

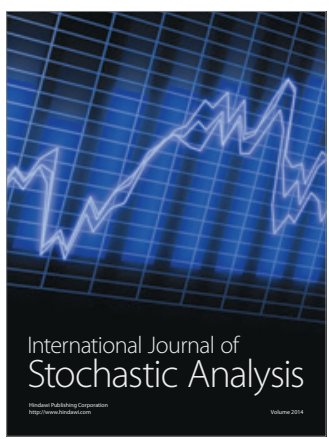

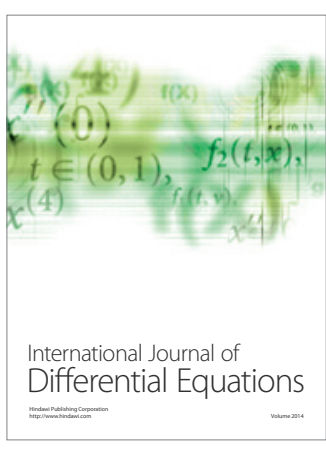
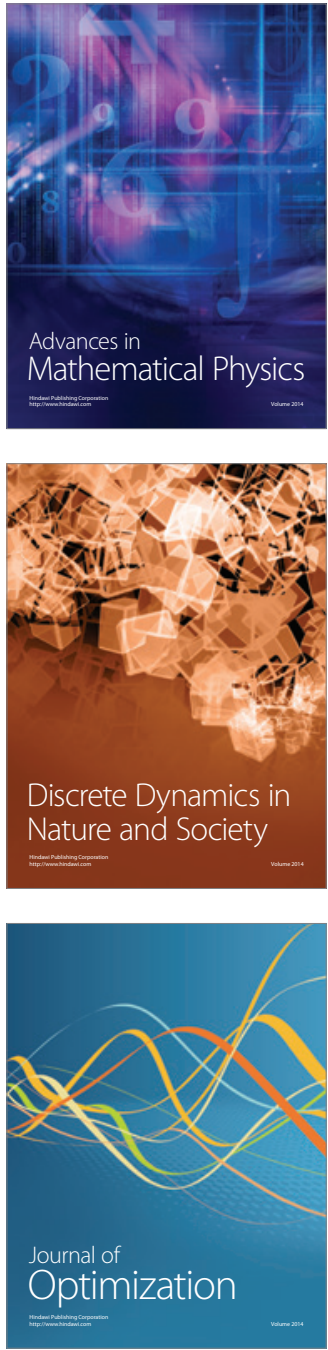